\title{
Hypersurfaces compactes d'un fibré vectoriel Riemannien à courbure moyenne prescrite
}

\author{
Pascal CHERRIER ${ }^{1}$ \\ et
}

Abdellah HANANI ${ }^{2}$

\begin{abstract}
Let $M$ be a compact Riemannian manifold without boundary and let $E$ be a Riemannian vector bundle over $M$. If $\Sigma$ denotes the sphere subbundle of $E$, we look for embeddings of $\Sigma$ into $E$ admitting a prescribed mean curvature.
\end{abstract}

Mots clés : Connexions, relèvement, courbure moyenne, estimées a priori, les méthodes. Classification matières $2010: 35 \mathrm{~J} 60$, 53C55, 58G30.

\section{Introduction}

Dans cette étude, on désigne par $(M, g)$ une variété Riemannienne compacte sans bord, de dimension $n \geq 1$ et $(E, \tilde{g})$ un fibré vectoriel Riemannien sur $M$ de rang $m \geq 2$. On note $\Sigma$ le fibré unitaire correspondant et $E_{*}$ le fibré $E$ privé de la section nulle. On s'intéresse à la mise en évidence d'une hypersurface de $E_{*}$ admettant une courbure moyenne égale à $K$, une fonction $\mathscr{C}^{\infty}$ strictement positive donnée sur $E_{*}$, et définie comme la trace de la seconde forme fondamentale relativement à la métrique. C'est un problème global où il s'agit de déterminer un plongement $\mathcal{Y}$ de $\Sigma$ dans $E_{*}$ ayant une courbure moyenne prescrite.

Dans le cadre euclidien, c'est-à-dire quand $M$ est réduite à un point, un théorème de Bakelman et Kantor [2] assure, en dimension 3, l'existence d'une telle hypersurface sous la condition que la fonction $K$ décroît plus vite que la courbure moyenne de sphères concentriques, i.e. il existe deux réels $r_{1}$ et $r_{2}$ tels que $0<r_{1} \leq 1 \leq r_{2}$ et

$$
K(\xi)>\frac{m-1}{\|\xi\|} \text { si }\|\xi\|<r_{1}, K(\xi)<\frac{m-1}{\|\xi\|} \text { si }\|\xi\|>r_{2}
$$

jointe à l'hypothèse de monotonicité

$$
\frac{\partial[r K(r \xi)]}{\partial r} \leq 0, \text { pour tout } \xi \in \Sigma .
$$

1. Current address : Université de Paris VI, UFR 920 de Mathématiques, B.C. 172, 4 place Jussieu, 75252 Paris Cedex 05, France

E-mail address : cherrier@ccr.jussieu.fr

2. Current address : Université de Lille 1, UFR de Mathématiques, Bât. M2, 59655, Villeneuve d'Ascq Cedex, France

E-mail address : abdellah.hanani@math.univ-lille1.fr 
Une autre preuve, valable en toute dimension, est donnée par Treibergs et Wei [8] sous les conditions précédentes. L'hypothèse (1.2) leur a permis d'appliquer la méthode de continuité et leur donne l'unicité à homothétie près.

Dans le cadre des fibrés envisagés ici, des méthodes différentes de celles des auteurs précités s'imposent. En effet, on ramène le problème à la résolution d'une équation elliptique sur $\Sigma$ dans laquelle, et contrairement aux cas ci-dessus cités, les dérivées de la fonction inconnue $u$ sont pondérées par des puissances de $\mathrm{e}^{u}$ dont le degré varie de 0 à 4 . Ceci complique substantiellement l'obtention de l'estimée $\mathscr{C}^{1}$ et met en péril l'unicité à homothétie près même sous une hypothèse du type (1.2).

Quand la fonction prescrite ne dépend que du rayon, on obtient la caractérisation suivante.

Théorème 1. Soit $K$ une fonction continue sur $E_{*}$, partout strictement positive, et telle que $K(\xi)=K(\|\xi\|)$ pour tout $\xi \in E_{*}$. Alors, il existe un graphe radial $\mathcal{Y}$ sur $\Sigma$, de classe $\mathscr{C}^{\infty}$, à courbure moyenne égale à $K$ si et seulement s'il existe un réel $r>0$ tel qu'on ait:

$$
K(r \xi)=(m-1) r^{-1} \text { quel que soit } \xi \in \Sigma .
$$

La suffisance dans ce résultat est une conséquence de l'expression de l'opérateur de Weingarten de l'hypersurface $\Sigma_{r}$, le fibré en sphère de rayon $r>0$. Quant à la nécéssité, elle ne requiert que la continuité de $K$. On généralise ce résultat comme suit.

Théorème 2. Soit $K \in \mathscr{C}^{\infty}\left(E_{*}\right)$ une fonction partout strictement positive. On fait l'hypothèse qu'il existe deux réels $r_{1}$ et $r_{2}, 0<r_{1} \leq 1 \leq r_{2}$, tels que les inégalités (1.1) soient satisfaites. Notons $\Sigma_{r_{1}, r_{2}}=\left\{\xi \in E ; r_{1} \leq \| \xi \mid \leq r_{2}\right\}$ et supposons que $K$ vérifie (1.2) en tout point de $\Sigma_{r_{1}, r_{2}}$ et que la composante horizontale de son gradient y est partout nulle. Il existe alors un graphe radial $\mathcal{Y}$ sur $\Sigma$ dont la courbure moyenne est donnée par $K$. Celui-ci étant de la forme $\xi \in \Sigma \mapsto e^{u(\xi)} \xi$, où $u \in \mathscr{C}^{\infty}(\Sigma)$ est une fonction à gradient horizontal identiquement nul.

Dans le but d'établir ce théorème, on constuit le graphe $\mathcal{Y}$, qui peut être vu comme une fibration dont la fibre $\mathcal{Y}_{x}$ au dessus du point $x \in M$ est une hypersurface de $E_{x}$ obtenue par projection radiale de la fibre $\Sigma_{x}$ de $\Sigma$, en résolvant une équation aux dérivées partielles elliptique sur $\Sigma$. Pour résoudre cette dernière, on utilise l'invariance du degré de Leray-Schauder d'une homotopie compacte relativement à un domaine borné du sousensemble des fonctions de classe $\mathscr{C}^{1, \alpha}(\Sigma)$ dont la composante horizontale du gradient est identiquement nulle. L'application de celle-ci repose en partie sur l'obtention d'une estimée a priori dans $\mathscr{C}^{1, \alpha}(\Sigma)$. Remarquons que, dans ce résultat, l'hypothèse (1.2) est faite pour assurer la nullité du gradient horizontal de la solution. Celle-ci peut être omise. A cet égard, on démontre le théorème suivant.

Théorème 3. Soit $K \in \mathscr{C}^{\infty}\left(E_{*}\right)$ une fonction partout strictement positive. On suppose qu'il existe deux réels $r_{1}$ et $r_{2}, 0<r_{1} \leq 1 \leq r_{2}$, tels que les inégalités (1.1) soient satisfaites. Il existe alors un graphe radial $\mathcal{Y}$ sur $\Sigma$, de classe $\mathscr{C}^{\infty}$, dont la courbure moyenne est donnée par $K$, et tel que $r_{1} \leq\|\xi\| \leq r_{2}$ pour tout $\xi \in \mathcal{Y}$. 
Pour prouver l'existence d'une solution sous ces conditions, on se place dans le cadre fonctionnel $\mathscr{C}^{\infty}$ et on applique alors le théorème de point fixe de Nagumo [7]. Ceci est due au fait qu'en vue d'établir l'estimée $\mathscr{C}^{1}$ nécéssaire pour résoudre, il faut disposer auparavant d'un contrôle sur le laplacien horizontal de toute solution éventuelle. Dans ce but, on étend l'équation à résoudre au voisinage de $\Sigma$, les valeurs à prescrire au bord étant du type Neumann. L'équation à considérer au voisinage de $\Sigma$ est de sorte qu'une dérivation radiale et restriction à $\Sigma$ de celle-ci donne le contrôle souhaité.

Nous présentons cet article en quatre parties. A la dernière, on donne la preuve des théorèmes et une remarque montrant que l'hypothèse de croissance des théorèmes 2 et 3 est dans un certain sens la meilleure possible. L'estimée a priori de la norme $\mathscr{C}^{1}$ nécéssaire pour résoudre est présentée à la troisième partie. Une mise en équation est donnée à la seconde partie et, pour plus de clarté, on consacre la première partie de cet article à des notations et rappels préliminaires.

\section{Notations et préliminaires}

1- Soit $(M, g)$ une variété Riemannienne compacte sans bord de dimension $n \geq 1$. Soient $(E, \tilde{g})$ un fibré vectoriel Riemannien sur $M$ de rang $m \geq 2, \pi$ la projection naturelle de $E$ sur $M$ et $E_{*}$ le fibré $E$ privé de la section nulle. On note $\nabla$ la connexion de Levi-Civita de la variété $(M, g)$ et $\tilde{\nabla}$ une connexion métrique sur le fibré $(E, \tilde{g})$.

Soit $U$ un ouvert de $M$, domaine du système de coordonnées $\left(x^{i}\right)_{1 \leq i \leq n}$ sur $M$, et au dessus duquel $E$ est trivial. Notons $\varepsilon_{i}=\frac{\partial}{\partial x^{i}}, 1 \leq i \leq n$, et considérons un repère de sections de $E$ au dessus de $U$ noté $\left(\varepsilon_{\alpha}\right), n+1 \leq \alpha \leq n+m$. Si $\xi \in \pi^{-1}(U)$ et $x=\pi(\xi)$, on écrit $\xi=y^{\alpha} \varepsilon_{\alpha}(x) ;\left(x^{i}, y^{\alpha}\right)_{i, \alpha}$ est alors un système de coordonnées sur $\pi^{-1}(U)$.

Soient $x \in U, \xi=\xi^{\alpha} \varepsilon_{\alpha} \in \pi^{-1}(x)$ et $X=X^{i} \varepsilon_{i}$ un champ de vecteurs tangents à $U$. Le relèvement horizontal de $X_{\mid x}$ en $\xi$ est l'élément $X^{H}(\xi) \in T_{\xi} E$ défini par

$$
X^{H}(\xi)=X^{i}(x)\left(\frac{\partial}{\left.\partial x^{i}\right|_{\xi}}-\xi^{\alpha} \Gamma_{i \alpha}^{\beta} \frac{\partial}{\left.\partial y^{\beta}\right|_{\xi}}\right),
$$

où les $\Gamma_{i \alpha}^{\beta}, i \in\{1, \ldots, n\}$ et $\alpha, \beta \in\{n+1, \ldots, n+m\}$ désigent les symboles de Christoffel de la connexion $\tilde{\nabla}$ dans le système de coordonnées $\left(x^{i}, y^{\alpha}\right)$.

Sur l'ouvert $\pi^{-1}(U)$, on considère alors le repère mobile

$$
\mathcal{S}=\left\{e_{i}, e_{\alpha} / i=1, \ldots, n \text { et } \alpha=n+1, \ldots, n+m\right\},
$$

où $e_{\alpha}=\frac{\partial}{\partial y^{\alpha}}$ et $e_{i}$ est le relèvement horizontal de $\varepsilon_{i}$. Ainsi

$$
e_{i}=\frac{\partial}{\partial x^{i}}-y^{\alpha} \Gamma_{i \alpha}^{\beta} \frac{\partial}{\partial y^{\beta}}
$$

On définit sur la variété $E$ une métrique Riemannienne $G$ en posant

$$
G\left(e_{i}, e_{j}\right)=g\left(\epsilon_{i}, \epsilon_{j}\right), \quad G\left(e_{\alpha}, e_{\beta}\right)=\tilde{g}\left(\epsilon_{\alpha}, \epsilon_{\beta}\right), \quad G\left(e_{i}, e_{\alpha}\right)=0 .
$$


On dispose alors, sur la variété $E$, de la connexion de Levi-Civita associée à la métrique $G$ et de la connexion $D$ de Sasaki [9] et [10] définie par

$$
D_{e_{i}} e_{j}=\Gamma_{i j}^{k} e_{k}, D_{e_{i}} e_{\alpha}=\Gamma_{i \alpha}^{\beta} e_{\beta}, D_{e_{\alpha}} e_{i}=D_{e_{\alpha}} e_{\beta}=0,
$$

où les $\Gamma_{i j}^{k}, i, j, k \in\{1, \ldots, n\}$, désignent les symboles de Christoffel de la connexion $\nabla$ dans le système de coordonnées $\left(x^{i}\right)$. La connexion $D$ est $G$-métrique et ne coïncide pas avec la connexion de Levi-Civita de $G$; la torsion $T$ de $D$ est non nulle. En effet, notant $\mathbf{S}$ le tenseur de courbure de $\tilde{\nabla}$, les composantes de $T$ dans le repère mobile $\mathcal{S}$ sont toutes nulles sauf

$$
T_{i j}^{\alpha}=-y^{\beta} S_{\beta i j}^{\alpha}
$$

Les composantes dans $\mathcal{S}$ du tenseur de courbure $\mathcal{R}$ de $D$ sont données par

$$
R_{d c a b}=G\left(\left(D_{e_{a} e_{b}}-D_{e_{b} e_{a}}-D_{\left[e_{a}, e_{b}\right]}\right) e_{c}, e_{d}\right)
$$

et

$$
R_{c a b}^{d}=G^{d e} R_{e c a b} .
$$

Par un calcul direct, on montre qu'en particulier, pour $1 \leq i, j \leq n$ et $n+1 \leq \alpha, \beta, \lambda, \mu \leq$ $n+m$, on a :

$$
R_{\alpha \beta j}^{i}=R_{\alpha \beta j}^{\lambda}=R_{\alpha \beta \mu}^{i}=R_{\alpha \beta \mu}^{\lambda} \equiv 0 .
$$

2- On note $\Sigma=\{\xi \in E /\|\xi\|=1\}$ le sous-fibré unitaire de $E, \pi_{1}$ la surjection naturelle du fibré $\Sigma$ sur $M, r$ la fonction $r(\xi)=\|\xi\|$ et $\nu$ le champ radial unitaire. Sur l'ouvert $\pi^{-1}(U)$ muni des coordonnées $\left(x^{i}, y^{\alpha}\right), 1 \leq i \leq n$ et $n+1 \leq \alpha \leq n+m$, le champ $\nu$ est donné par

$$
\nu=r^{-1} y^{\alpha} \frac{\partial}{\partial y^{\alpha}}
$$

et l'ouvert $\pi_{1}^{-1}(U)$ de $\Sigma$ est donné, comme sous-variété de dimension $n+m-1$ de $\pi^{-1}(U)$, par

$$
\tilde{g}_{\alpha \beta} y^{\alpha} y^{\beta}=1 .
$$

Ce qui, par un calcul direct montre que le champ radial unitaire $\nu$ est normal à $\Sigma$. De l'expression de $\nu$, celle du relèvement horizontal d'un vecteur $X \in T_{x} M$ au point $\xi=\left(x, y^{\alpha}\right) \in \Sigma$, donnée par (2.2), et de la définition de la métrique $G$, on voit que l'espace tangent à $\Sigma$ au point $\xi$ est une somme directe du sous-espace horizontal $H_{\xi} E$ de $T_{\xi} E$ et de l'espace tangent à la fibre de $\Sigma$ passant par $\xi$.

Dans tout ce qui suit, le paramètre $\mu_{a}$ sera égal à 0 ou 1 selon que la direction $a$ est verticale ou horizontale. Fixons un repère mobile tangent à $E$ de la forme

$$
\mathcal{R}=\left\{e_{i}, e_{\alpha} / i=1, \ldots, n \text { et } \alpha=n+1, \ldots, n+m\right\},
$$

où les $e_{i}$ sont des champs de vecteurs horizontaux obtenus par relèvement horizontal d'un repère mobile $\left(\epsilon_{i}\right)_{1 \leq i \leq n}$ sur $M$ et où les $e_{\alpha}$ sont des champs de vecteurs verticaux avec $e_{n+m}=\nu$. On notera

$$
\mathcal{R}^{*}=\left\{\omega^{A}, A \leq n+m\right\}
$$


le corepère dual de $\mathcal{R}$. La restriction à $\Sigma$ des champs $e_{a}$, pour $a \leq n+m-1$, constitue un repère mobile $\mathcal{R}_{1}$ tangent à $\Sigma$ et

$$
\mathcal{R}_{1}^{*}=\left\{\sigma^{a}, a \leq n+m-1\right\},
$$

où $\sigma^{a}$ est l'image inverse de $\omega^{a}$ par $i$, injection canonique de $\Sigma$ dans $E$, n'est autre que le corepère dual de $\mathcal{R}_{1}$.

Appliquons $D$ à $e_{a}$, le résultat est une 1-forme sur $E$ à valeurs dans $T E$ dont l'expression dans la repère $\mathcal{R}$ nous permet d'introduire la matrice $\left(\omega_{B}^{A}\right)$ de 1-formes définie par les égalités

$$
D e_{A}=\omega_{A}^{B} \otimes e_{B}
$$

Du fait que $\nu$ est unitaire et puisque $D$ est $G$-métrique, on voit que

$$
G\left(D_{e_{a}} \nu, \nu\right)=0, \text { pour } 1 \leq a \leq n+m .
$$

On en déduit que, sur $\Sigma_{r}$, le fibré en sphères de rayon $r$,

$$
\omega_{n+m}^{n+m}=0 .
$$

D'autre part, si $X$ est un champ de vecteurs verticaux tangents à $\Sigma_{r}$ et donc vérifiant $i_{*} X r=0$ sur $\Sigma_{r}$, on aura

$$
D_{i_{*} X} \nu=r^{-1}\left(i_{*} X . y^{\alpha}\right) \frac{\partial}{\partial y^{\alpha}}+r^{-1} y^{\alpha} D_{i_{*} X}\left(\frac{\partial}{\partial y^{\alpha}}\right)=r^{-1} i_{*} X .
$$

Eu égard à (2.4) qui implique que $D_{i_{*} X}\left(\frac{\partial}{\partial y^{\alpha}}\right)=0$. De même, compte tenu de la définition (2.4) de la connexion $D$ et de l'expression (2.2) du relèvement horizontal d'un champ de vecteurs, si dans $U, \varepsilon_{i}=\varepsilon_{i}^{j} \frac{\partial}{\partial x^{j}}$ et $e_{i}=\left(\varepsilon_{i}\right)^{H}$, on voit que

$$
D_{e_{j}} \nu=r^{-1} \varepsilon_{j}^{h}\left(\frac{\partial y^{\alpha}}{\partial x^{h}}-y^{\lambda} \Gamma_{h \lambda}^{\mu} \frac{\partial y^{\alpha}}{\partial y^{\mu}}\right) \frac{\partial}{\partial y^{\alpha}}+r^{-1} y^{\alpha} \varepsilon_{j}^{h} \Gamma_{h \alpha}^{\mu} \frac{\partial}{\partial y^{\mu}} .
$$

D'où

$$
D_{e_{j}} \nu=r^{-1} \epsilon_{j}^{h}\left[-y^{\lambda} \Gamma_{h \lambda}^{\mu} \delta_{\mu}^{\alpha} \frac{\partial}{\partial y^{\alpha}}+y^{\alpha} \Gamma_{h \alpha}^{\mu} \frac{\partial}{\partial y^{\mu}}\right]=0 .
$$

Ainsi, compte tenu de (2.9) et (2.10),

$$
D_{e_{a}} \nu=\left(1-\mu_{a}\right) r^{-1} e_{a} \quad \text { pour } \quad a \leq n+m-1 .
$$

Reportons dans (2.7), il en découle que, sur $\Sigma_{r}$, on a

$$
\omega_{n+m}^{a}=\left(1-\mu_{a}\right) r^{-1} \omega^{a} \text { pour } a \leq n+m-1
$$

et par suite, compte tenu de (2.8),

$$
D_{\nu} \nu=0
$$


D'autre part, la compatibilité de la connexion $D$ avec la métrique $G$ montre que

$$
G\left(D_{e_{b}} e_{a}, \nu\right)=-G\left(e_{a}, D_{e_{b}} \nu\right)
$$

ce qui, compte tenu de (2.12), implique que

$$
\omega_{a}^{n+m}\left(e_{b}\right)=-\left(1-\mu_{b}\right) r^{-1} G_{a b} \text { pour } a, b \leq n+m-1 .
$$

A présent, on donne les composantes du tenseur de courbure $\tilde{\mathcal{R}}$ de $\Sigma$. Utilisons l'équation de Gauss et (2.14) on voit que

$$
\tilde{R}_{d c a b}=R_{d c a b}+\left(1-\mu_{a}\right)\left(1-\mu_{b}\right)\left(G_{a d} G_{b c}-G_{a c} G_{b d}\right) .
$$

Ainsi, tenons compte de (2.6), nous obtenons la valeur des composantes qui seront utilisées ultérieurement :

$$
\tilde{R}_{\alpha \beta \gamma}^{j}=\tilde{R}_{\alpha \beta i}^{j}=\tilde{R}_{\alpha \beta i}^{\gamma}=0, n+1 \leq \alpha, \beta, \gamma \leq n+m-1 \text { et } 1 \leq i, j \leq n,
$$

et

$$
\tilde{R}_{\alpha \beta \mu}^{\lambda}=\delta_{\beta}^{\lambda} G_{\alpha \mu}-\delta_{\mu}^{\lambda} G_{\alpha \beta}, n+1 \leq \alpha, \beta, \lambda, \mu \leq n+m-1 .
$$

\section{Mise en équation}

Dans cette partie, on donnera l'équation de la courbure moyenne. On conserve toutes les notations du dernier paragraphe de la section précédente. Soit $u \in \mathscr{C}^{2}(\Sigma)$ une fonction qu'on prolonge à $E_{*}$ en la maintenant radialement constante. On considère l'application $\mathcal{Y}$ de $\Sigma$ dans $E$ telle que

$$
\mathcal{Y}(\xi)=e^{u(\xi)} \xi, \text { pour } \xi \in \Sigma .
$$

La différentielle $D \mathcal{Y}$ de $\mathcal{Y}$ est une section de $T^{*} \Sigma \otimes T E$ qu'on peut écrire sous la forme

$$
D \mathcal{Y}=\sigma^{a} \otimes E_{a} \text {, où pour } a \leq n+m-1, E_{a}=D \mathcal{Y}\left(e_{a}\right) \text {. }
$$

Les champs de vecteurs $\left\{E_{a}, a \leq n+m-1\right\}$ définissent un repère mobile tangent au graphe $\mathcal{Y}$. Un calcul direct donne

$$
E_{a}=e_{a}+e^{u} D_{a} u \nu
$$

et le champ unitaire normal à $\mathcal{Y}$ est donc

$$
\tilde{\nu}=f\left(\nu-e^{u} D^{a} u e_{a}\right), \quad f=\left(1+e^{2 u} D_{a} u D^{a} u\right)^{-\frac{1}{2}} .
$$

La métrique $H$ induite par $G$ sur $\mathcal{Y}$ est

$$
H=H_{a b} \sigma^{a} \otimes \sigma^{b} \text {, où } H_{a b}=G\left(E_{a}, E_{b}\right)=G_{a b}+e^{2 u} D_{a} u D_{b} u
$$

et la résolution de l'équation $H^{a b} H_{b c}=\delta_{c}^{a}$ donne les composantes contravariantes de celle-ci, on vérifie que :

$$
H^{a b}=G^{a b}-f^{2} e^{2 u} D^{a} u D^{b} u .
$$


Enfin, rappelons qu'avec le choix ci-dessus du champ normal unitaire $\tilde{\nu}$, la courbure moyenne $\mathcal{M}_{\mathcal{Y}}$ de $\mathcal{Y}$ est donnée par

$$
\mathcal{M}_{\mathcal{Y}}=H^{a b} G\left(D_{E_{a}} \tilde{\nu}, E_{b}\right) .
$$

Dans le corepère $\mathcal{R}^{*}$, la différentielle de la fonction $u$ est donné par

$$
d u=\sum_{a=1}^{n+m-1} D_{a} u \omega^{a} .
$$

La composante $D_{a} u$ est homogène de degré 0 ou -1 selon que la direction $a$ est horizontale ou verticale, i.e. $D_{a} u$ est homogène de degré $\left(\mu_{a}-1\right)$. De même, on a

$$
D_{a b} u=D^{2} u\left(e_{a}, e_{b}\right)=\left(D_{e_{a}} D u\right)\left(e_{b}\right) .
$$

D'où

$$
D_{a b} u=D_{e_{a}}\left(D u\left(e_{b}\right)\right)-D u\left(D_{e_{a}} e_{b}\right)
$$

et on vérifie que la composante $D_{a b} u$ est homogène de degré $\left(\mu_{a}+\mu_{b}-2\right)$. En particulier, $\operatorname{sur} \mathcal{Y}$, on peut écrire

$$
D_{a \nu} u=D_{e_{a}}(D u(\nu))-D u\left(D_{e_{a}} \nu\right)=-D u\left(D_{e_{a}} \nu\right) .
$$

La dernière égalité découle du fait que $u$ est une fonction radialement constante. La relation (2.11) implique alors que

$$
D_{a \nu} u=-\left(1-\mu_{a}\right) e^{-u} D_{a} u \text { pour } a \leq n+m-1 .
$$

Tenons compte de la relation (2.13), un calcul analogue montre que

$$
D_{\nu \nu} u=0
$$

A présent, de la nature du prolongement de $u$ qui implique que $D_{\nu} u=0$, et la relation (2.13) qui dit que $D_{\nu} \nu=0$, on voit que

$$
D_{E_{a}} E_{b}=D_{e_{a}}\left(e_{b}+e^{u} D_{b} u \nu\right)+e^{u} D_{a} D_{\nu} e_{b}+e^{2 u} D_{a} u\left(\nu \cdot D_{b} u\right) \nu
$$

Le fait que les composantes de la torsion de la forme $T_{n+m b}^{a}$ sont identiquement nulles et la relation (2.11) permettent d'écrire :

$$
D_{\nu} e_{b}=D_{e_{b}} \nu=(1-\mu b) e^{-u} e_{b} .
$$

D'autre part,

$$
D_{\nu}\left(D_{b} u\right)=D_{\nu b} u+D u\left(D_{\nu} e_{b}\right)=-\left(1-\mu_{b}\right) e^{-u} D_{b} u+D u\left(\left(1-\mu_{b}\right) e^{-u} e_{b}\right) .
$$

La dernière égalité est une conséquence de (3.1) et la relation ci-dessus. Ainsi, on voit que $D_{\nu}\left(D_{b} u\right)=0$ et donc

$$
D_{E_{a}} E_{b}=D_{e_{a}} e_{b}+e^{u}\left[D_{e_{a}}\left(D_{b} u\right)+D_{a} u D_{b} u\right] \nu+e^{u} D_{b} u D_{e_{a}} \nu+\left(1-\mu_{b}\right) D_{a} u e_{b} .
$$


Une égalité qui, compte tenu de (2.11), s'écrit sous la forme

$$
D_{E_{a}} E_{b}=D_{e_{a}} e_{b}+e^{u}\left[D_{e_{a}}\left(D_{b} u\right)+D_{a} u D_{b} u\right] \nu+D_{b} u\left(1-\mu_{a}\right) e_{a}+D_{a} u\left(1-\mu_{b}\right) e_{b}
$$

Or,

$$
D_{e_{a}} e_{b}=\sum_{1}^{n+m-1} \omega_{b}^{c}\left(e_{a}\right) e_{c}+\omega_{b}^{n+m}\left(e_{a}\right) \nu=\sum_{1}^{n+m-1} \omega_{b}^{c}\left(e_{a}\right) e_{c}-\left(1-\mu_{a}\right) e^{-u} G_{a b} \nu
$$

Donc, tenons compte de (2.14), on obtient

$$
D_{e_{a}} e_{b}=\sum_{1}^{n+m-1} \omega_{b}^{c}\left(e_{a}\right) e_{c}-\left(1-\mu_{a}\right) e^{-u} G_{a b} \nu
$$

Par suite, la définition de la dérivée covariante permet d'écrire

$$
D_{e_{a}}\left(D_{b} u\right)=D_{a b} u+D u\left(D_{e_{a}} e_{b}\right)=D_{a b} u+\sum_{1}^{n+m-1} \omega_{b}^{c}\left(e_{a}\right) D_{c} u .
$$

De sorte que

$$
D_{e_{a}} e_{b}+e^{u}\left(e_{a} D_{b} u\right) \nu=\sum_{1}^{n+m-1} \omega_{b}^{c}\left(e_{a}\right) E_{c}+e^{-u}\left[e^{2 u} D_{a b} u-\left(1-\mu_{a}\right) G_{a b}\right] \nu
$$

Reportons dans (3.3), compte tenu du fait que $E_{a}=e_{a}+e^{u} D_{a} u \nu$, la relation qui en résulte peut s'écrire sous la forme :

$$
\begin{aligned}
D_{E_{a}} E_{b}= & \sum_{1}^{n+m-1} \omega_{b}^{c}\left(e_{a}\right) E_{c}+D_{b} u\left(1-\mu_{a}\right) E_{a}+D_{a} u\left(1-\mu_{b}\right) E_{b} \\
& +e^{-u}\left[-\left(1-\mu_{a}\right) H_{a b}+\mu_{b} e^{2 u} D_{a} u D_{b} u+e^{2 u} D_{a b} u\right] \nu
\end{aligned}
$$

et par suite, eu égard au fait que $G\left(E_{c}, \tilde{\nu}\right)=0$ pour $c \leq n+m-1$, on obtient :

$$
G\left(D_{E_{a}} E_{b}, \tilde{\nu}\right)=f e^{-u}\left[-\left(1-\mu_{a}\right) H_{a b}+\mu_{b} e^{2 u} D_{a} u D_{b} u+e^{2 u} D_{a b} u\right] .
$$

Ainsi et puisque $G\left(D_{E_{a}} \tilde{\nu}, E_{b}\right)=-G\left(D_{E_{a}} E_{b}, \tilde{\nu}\right)$, on trouve que

$$
f^{-1} e^{u} \mathcal{M}_{\mathcal{Y}}=H^{a b}\left[\left(1-\mu_{a}\right) H_{a b}-\mu_{b} e^{2 u} D_{a} u D_{b} u-e^{2 u} D_{a b} u\right] .
$$

Prenons l'image inverse sur $\Sigma$ de cette équation, tenons compte de l'homogenéité des dérivées covariantes de $u$ et notons $\tilde{D}^{a} u=e^{\mu_{a} u} D^{a} u$ et $\tilde{D}^{a b} u=e^{\left(\mu_{a}+\mu_{b}\right) u} D^{a b} u$, on obtient

$$
A^{a b}(u) \tilde{D}_{a b} u=-v_{2}+(m-1)(1+v)-(1+v)^{\frac{3}{2}} e^{u} \mathcal{M}_{\mathcal{Y}}\left(e^{u} \xi\right),
$$

où $A^{a b}(u)=(1+v) G^{a b}-\tilde{D}^{a} u \tilde{D}^{b} u$ et

$$
v=v_{1}+v_{2}, v_{1}=\left(1-\mu_{a}\right) \tilde{D}_{a} u \tilde{D}^{a} u, v_{2}=\mu_{a} \tilde{D}_{a} u \tilde{D}^{a} u .
$$

Ainsi, la recherche d'un graphe radial à courbure moyenne prescrite $K$ revient à résoudre sur $\Sigma$ l'équation elliptique suivante :

$$
A^{a b}(u) \tilde{D}_{a b} u=-v_{2}+(m-1)(1+v)-(1+v)^{\frac{3}{2}} e^{u} K\left(e^{u} \xi\right) .
$$




\section{Estimations a priori}

Lemme 1. Soient $K \in \mathscr{C}^{\infty}\left(E_{*}\right)$ une fonction partout strictement positive et $u \in \mathscr{C}^{3}(\Sigma)$ une solution de l'équation

$$
A^{a b} \tilde{D}_{a b} u=-v_{2}+(m-1)(1+v)-(1+v)^{\frac{3}{2}} e^{u} K\left(e^{u} \xi\right) \equiv F
$$

telle qu'il existe deux réels strictement positifs $r_{1}$ et $r_{2}$ vérifiant $r_{1} \leq e^{u} \leq r_{2}$. Notons $\Sigma_{r_{1}, r_{2}}=\left\{\xi \in E ; r_{1} \leq\|\xi\| \leq r_{2}\right\}$ et $r$ la fonction $r(\xi)=\|\xi\|$, et supposons qu'en tout point de $\Sigma$ où $v \geq 1$, on ait

$$
\left(\mu_{a}+\mu_{b}\right) G^{a b} \tilde{D}_{a b} u \leq-\alpha \sqrt{v}
$$

où l'on a noté

$$
\alpha=2 \sup _{\xi \in \Sigma}\left[\sup _{\Sigma_{r_{1}, r_{2}}}\left|r \frac{\partial[r K(r \xi)]}{\partial r}\right|\right] .
$$

Alors il existe une constante positive $C_{1}$ ne dépendant que de $(M, g),(E, \tilde{g}), r_{1}, r_{2}, \max _{\Sigma_{r_{1}, r_{2}}} K$ et $\|K\|_{\mathscr{C}^{1}\left(\Sigma_{\left.r_{1}, r_{2}\right)}\right.}$ telle que $|D u| \leq C_{1}$ partout dans $\Sigma$.

Démonstration. Soient $u \in \mathscr{C}^{3}(\Sigma)$ une solution de (4.1), $l$ un réel strictement positif fixé ultérieurement et $\Gamma$ la fonctionnelle définie, sur $\Sigma$, par

$$
\Gamma(u)=(1+v) e^{-l u} .
$$

En un point $\xi \in \Sigma$ où $\Gamma(u)$ atteint son maximum, on a :

$$
\frac{D_{a} \Gamma}{\Gamma}=\frac{D_{a} v}{1+v}-l D_{a} u=0
$$

et

$$
A^{a b} \tilde{D}_{a b} \Gamma \leq 0
$$

c'est-à-dire, tenons compte de (4.1),

$$
A^{a b} \tilde{D}_{a b} v-\frac{A^{a b} \tilde{D}_{a} v \tilde{D}_{b} v}{1+v}-l(1+v) F \leq 0 .
$$

Or,

$$
\begin{gathered}
A^{a b} \tilde{D}_{a b} v=4 v_{2} A^{a b} \tilde{D}_{a} u \tilde{D}_{b} u+2 v_{2} A^{a b} \tilde{D}_{a b} u+8 A^{a b} \mu_{c} \tilde{D}_{a c} u \tilde{D}^{c} u \tilde{D}_{b} u \\
+2 A^{a b} \tilde{D}_{a}^{c} u \tilde{D}_{b c} u+2 A^{a b} \tilde{D}_{a b c} u \tilde{D}^{c} u
\end{gathered}
$$

où l'on a noté $\tilde{D}_{a b c} u=e^{\left(\mu_{a}+\mu_{b}+\mu_{c}\right) u} D_{a b c} u$. Une permutation de l'ordre des indices de dérivation covariante dans le terme des dérivées troisièmes, celle-ci gènère des termes en torsion et en courbure, montre que

$$
\begin{aligned}
& A^{a b} \tilde{D}_{a b} v=4 v_{2} v+2 v_{2} F+8 A^{a b} \mu_{c} \tilde{D}_{a c} u \tilde{D}^{c} u \tilde{D}_{b} u \\
& +2 A^{a b} \tilde{D}_{a}^{c} u \tilde{D}_{b c} u+2 A^{a b} \tilde{D}_{c a b} u \tilde{D}^{c} u+4 E_{1}+E_{2},
\end{aligned}
$$


où les termes $E_{1}$ et $E_{2}$ sont donnés par

$$
E_{1}=-A^{a b} e^{\left(\mu_{a}+\mu_{b}+\mu_{c}\right) u} T_{a c}^{d} D_{b d} u \tilde{D}^{c} u
$$

et

$$
E_{2}=-2 A^{a b} e^{\left(\mu_{a}+\mu_{b}+\mu_{c}\right) u}\left(\tilde{R}_{b a c}^{d}+D_{a} T_{b c}^{d}-T_{a c}^{h} T_{h b}^{d}\right) D_{d} u \tilde{D}^{c} u .
$$

A présent, on dérive une fois l'équation (4.1), il vient

$$
A^{a b} \tilde{D}_{c a b} u+\left(\mu_{a}+\mu_{b}\right) A^{a b} \tilde{D}_{a b} u \tilde{D}_{c} u+\left(\tilde{D}_{c} A^{a b}\right) \tilde{D}_{a b} u=\tilde{D}_{c} F .
$$

Développons $\left(\tilde{D}_{c} A^{a b}\right)$ et saturons par $\tilde{D}^{c} u$, on obtient :

$$
\begin{gathered}
A^{a b} \tilde{D}_{c a b} u \tilde{D}^{c} u=\tilde{D}_{c} F \tilde{D}^{c} u-v\left(\mu_{a}+\mu_{b}\right) A^{a b} \tilde{D}_{a b} u-\tilde{D}_{c} v \tilde{D}^{c} u G^{a b} \tilde{D}_{a b} u \\
+v\left(\mu_{a}+\mu_{b}\right) \tilde{D}^{a} u \tilde{D}^{b} u \tilde{D}_{a b} u+\tilde{D}_{c}{ }^{a} u \tilde{D}^{c} u \tilde{D}^{b} u \tilde{D}_{a b} u+\tilde{D}^{a} u \tilde{D}^{c} u \tilde{D}_{c}{ }^{b} u \tilde{D}_{a b} u .
\end{gathered}
$$

D'après (4.1), on peut écrire $G^{a b} \tilde{D}_{a b} u=\frac{F+\tilde{D}^{a} u \tilde{D}^{b} u \tilde{D}_{a b} u}{1+v}$. Il s'en suit que

$$
\tilde{D}_{c} v \tilde{D}^{c} u \tilde{D}_{a}^{a} u=\frac{F}{1+v} \tilde{D}_{c} v \tilde{D}^{c} u+\frac{\tilde{D}^{a} u \tilde{D}^{b} u \tilde{D}_{a b} u \tilde{D}_{c} v \tilde{D}^{c} u}{1+v} .
$$

Développons $\tilde{D}_{c} v$ dans le second terme du membre de droite, il vient

$$
\tilde{D}_{c} v \tilde{D}^{c} u \tilde{D}_{a}^{a} u=\frac{F \tilde{D}_{c} v \tilde{D}^{c} u}{1+v}+\frac{2\left(\tilde{D}^{a} u \tilde{D}^{b} u \tilde{D}_{a b} u\right)^{2}}{1+v}+\frac{2 v_{2} v}{1+v} \tilde{D}^{a} u \tilde{D}^{b} u \tilde{D}_{a b} u .
$$

Or

$$
\tilde{D}_{a} v=2 \tilde{D}^{b} u \tilde{D}_{a b} u+2 v_{2} \tilde{D}_{a} u
$$

Il en découle que

$$
\begin{aligned}
\tilde{D}_{c} v \tilde{D}^{c} u G^{a b} \tilde{D}_{a b} u & =\frac{F}{1+v} \tilde{D}_{c} v \tilde{D}^{c} u+\frac{2}{1+v}\left(\tilde{D}^{a} u \tilde{D}^{b} u \tilde{D}_{a b} u\right)^{2} \\
+ & \frac{v v_{2}}{1+v} \tilde{D}^{a} v \tilde{D}_{a} u-\frac{2 v\left(v_{2}\right)^{2}}{1+v} .
\end{aligned}
$$

D'autre part, la définition des composantes $A^{a b}$ montre que

$$
G^{a b}=\frac{A^{a b}+\tilde{D}^{a} u \tilde{D}^{b} u}{1+v} .
$$

De ce fait, la somme des deux derniers termes du membre de droite de (4.8) s'écrit sous la forme :

$$
\begin{gathered}
\tilde{D}_{c}{ }^{a} u \tilde{D}^{c} u \tilde{D}^{b} u \tilde{D}_{a b} u+\tilde{D}^{a} u \tilde{D}^{c} u \tilde{D}_{c}{ }^{b} u \tilde{D}_{a b} u=\frac{2\left(\tilde{D}^{a} u \tilde{D}^{b} u \tilde{D}_{a b} u\right)^{2}}{1+v} \\
+\frac{A^{a d} \tilde{D}_{c d} u \tilde{D}^{c} u \tilde{D}^{b} u \tilde{D}_{a b} u}{1+v}+\frac{A^{a d} \tilde{D}_{c d} u \tilde{D}^{c} u \tilde{D}^{b} u \tilde{D}_{b a} u}{1+v}
\end{gathered}
$$


Or, un simple calcul nous permet d'établir la commutation suivante :

$$
D_{c d} u=D_{d c} u-T_{c d}^{e} D_{e} u
$$

Il en découle que

$$
\begin{gathered}
\tilde{D}_{c}{ }^{a} u \tilde{D}^{c} u \tilde{D}^{b} u \tilde{D}_{a b} u+\tilde{D}^{a} u \tilde{D}^{c} u \tilde{D}_{c}{ }^{b} u \tilde{D}_{a b} u=\frac{2\left(\tilde{D}^{a} u \tilde{D}^{b} u \tilde{D}_{a b} u\right)^{2}}{1+v} \\
\left.+\frac{2 A^{a b}\left(\tilde{D}_{a c} u \tilde{D}^{c} u\right)\left(\tilde{D}_{b d} u^{d}\right.}{\tilde{D}} u\right) 1+v+E_{3}+E_{4},
\end{gathered}
$$

où l'on a noté

$$
E_{3}=-\frac{3}{1+v} A^{a b} e^{\left(\mu_{a}+\mu_{c}\right) u} T_{c a}^{e} D_{e} u \tilde{D}^{c} u \tilde{D}^{d} u \tilde{D}_{b d} u
$$

et

$$
E_{4}=\frac{1}{1+v} A^{a b} e^{\left(\mu_{a}+\mu_{b}+\mu_{c}+\mu_{d}\right) u} T_{c b}^{e} T_{d a}^{f} D_{e} u D_{f} u \tilde{D}^{c} u \tilde{D}^{d} u
$$

Ainsi, tenons compte de (4.9), on vérifie que

$$
\begin{gathered}
\tilde{D}_{c}{ }^{a} u \tilde{D}^{c} u \tilde{D}^{b} u \tilde{D}_{a b} u+\tilde{D}^{a} u \tilde{D}^{c} u \tilde{D}_{c}{ }^{b} u \tilde{D}_{a b} u=\frac{2\left(\tilde{D}^{a} u \tilde{D}^{b} u \tilde{D}_{a b} u\right)^{2}}{1+v} \\
+\frac{A^{a b} \tilde{D}_{a} v \tilde{D}_{b} v}{2(1+v)}+\frac{2\left(v_{2}\right)^{2} v}{1+v}-\frac{2 v_{2}}{1+v} A^{a b} \tilde{D}_{a} v \tilde{D}_{b} u+E_{3}+E_{4} .
\end{gathered}
$$

D'autre part, utilisons à nouveau (4.9), on obtient la forme suivante pour le terme $E_{3}$ :

$$
\begin{aligned}
E_{3}= & -\frac{3}{2(1+v)} A^{a b} e^{\left(\mu_{a}+\mu_{c}\right) u} T_{c a}^{e} D_{e} u \tilde{D}^{c} u \tilde{D}_{b} v \\
& +\frac{3 v_{2}}{1+v} A^{a b} e^{\left(\mu_{a}+\mu_{c}\right) u} T_{c a}^{e} D_{e} u \tilde{D}^{c} u \tilde{D}_{b} u .
\end{aligned}
$$

Une forme, qui compte tenu de (4.5) et de la définition des composantes $A^{a b}$, permet de vérifier que

$$
E_{3}=\left(-\frac{3 l}{2}+\frac{3 v_{2}}{1+v}\right) e^{\left(\mu_{a}+\mu_{c}\right) u} T_{c a}^{e} D_{e} u \tilde{D}^{c} u \tilde{D}^{a} u
$$

Reportons (4.10) et (4.14) dans (4.8), tenons compte à nouveau de la définition des composantes $A^{a b}$, on obtient :

$$
\begin{gathered}
A^{a b} \tilde{D}_{c a b} u \tilde{D}^{c} u=\frac{A^{a b} \tilde{D}_{a} v \tilde{D}_{b} v}{2(1+v)}+\tilde{D}_{c} F \tilde{D}^{c} u-\frac{F \tilde{D}_{a} v \tilde{D}^{a} u}{1+v}-\frac{v_{2}(2+v)}{1+v} \tilde{D}_{a} v \tilde{D}^{a} u \\
-v(1+v)\left(\mu_{a}+\mu_{b}\right) G^{a b} \tilde{D}_{a b} u+4 v \mu_{a} \tilde{D}^{a} u \tilde{D}^{b} u \tilde{D}_{a b} u+\frac{4 v\left(v_{2}\right)^{2}}{1+v}+E_{3}+E_{4} .
\end{gathered}
$$


Une égalité qui, compte tenu de (4.9), s'écrit sous la forme :

$$
\begin{gathered}
A^{a b} \tilde{D}_{c a b} u \tilde{D}^{c} u=\frac{A^{a b} \tilde{D}_{a} v \tilde{D}_{b} v}{2(1+v)}+\tilde{D}_{c} F \tilde{D}^{c} u-\frac{F \tilde{D}_{a} v \tilde{D}^{a} u}{1+v}-\frac{v_{2}(2+v)}{1+v} \tilde{D}_{a} v \tilde{D}^{a} u \\
-v(1+v)\left(\mu_{a}+\mu_{b}\right) G^{a b} \tilde{D}_{a b} u+2 v \mu_{a} \tilde{D}^{a} v \tilde{D}_{a} u-\frac{4\left(v v_{2}\right)^{2}}{1+v}+E_{3}+E_{4} .
\end{gathered}
$$

Multiplions cette relation par 2 et reportons dans (4.7), l'égalité qui en résulte s'écrit sous la forme :

$$
\begin{gathered}
A^{a b} \tilde{D}_{a b} v-\frac{A^{a b} \tilde{D}_{a} v \tilde{D}_{b} v}{(1+v)}=2 \tilde{D}_{a} F \tilde{D}^{a} u-2 \frac{F \tilde{D}_{a} v \tilde{D}^{a} u}{1+v}-\frac{2 v_{2}(2+v)}{1+v} \tilde{D}_{a} v \tilde{D}^{a} u \\
-2 v(1+v)\left(\mu_{a}+\mu_{b}\right) G^{a b} \tilde{D}_{a b} u+8 A^{a b} \mu_{c} \tilde{D}_{a c} u \tilde{D}^{c} u \tilde{D}_{b} u+4 v \mu_{a} \tilde{D}_{a} v \tilde{D}^{a} u \\
+2 v_{2} F+4 v v_{2}-\frac{8\left(v v_{2}\right)^{2}}{1+v}+2 A^{a b} \tilde{D}_{a}^{c} u \tilde{D}_{b c} u+4 E_{1}+E_{2}+2 E_{3}+2 E_{4} .
\end{gathered}
$$

La définition des composantes $A^{a b}$ montre, à nouveau, que

$$
2 \mu_{c} A^{a b} \tilde{D}_{a c} u \tilde{D}^{c} u \tilde{D}_{b} u=\left(\mu_{a}+\mu_{b}\right) \tilde{D}^{a} u \tilde{D}^{b} u \tilde{D}_{a b} u .
$$

D'autre part, d'après (4.9), on obtient :

$$
\left(\mu_{a}+\mu_{b}\right) \tilde{D}^{a} u \tilde{D}^{b} u \tilde{D}_{a b} u=2 \mu_{a} \tilde{D}^{a} u \tilde{D}^{b} u \tilde{D}_{a b} u=\mu_{a} \tilde{D}^{a} u \tilde{D}_{a} v-2\left(v_{2}\right)^{2} .
$$

Reportons dans (4.16), on obtient :

$$
\begin{gathered}
A^{a b} \tilde{D}_{a b} v-\frac{A^{a b} \tilde{D}_{a} v \tilde{D}_{b} v}{(1+v)}=2 \tilde{D}_{c} F \tilde{D}^{c} u-2 \frac{F \tilde{D}_{a} v \tilde{D}^{a} u}{1+v}-\frac{2 v_{2}(2+v)}{1+v} \tilde{D}_{a} v \tilde{D}^{a} u \\
-2 v(1+v)\left(\mu_{a}+\mu_{b}\right) G^{a b} \tilde{D}_{a b} u+4 v \mu_{a} \tilde{D}_{a} v \tilde{D}^{a} u+4 \mu_{a} \tilde{D}^{a} u \tilde{D}_{a} v+2 v_{2} F \\
+4 v_{2}\left(v_{1}-v_{2}\right)-\frac{8\left(v v_{2}\right)^{2}}{1+v}+2 A^{a b} \tilde{D}_{a}^{c} u \tilde{D}_{b c} u+4 E_{1}+E_{2}+2 E_{3}+2 E_{4}
\end{gathered}
$$

Supposons que

$$
v(\xi) \geq 1
$$

Tenons compte de (4.2) et (4.5), on voit que

$$
\begin{gathered}
A^{a b} \tilde{D}_{a b} v-\frac{A^{a b} \tilde{D}_{a} v \tilde{D}_{b} v}{(1+v)} \geq 2 \tilde{D}_{c} F \tilde{D}^{c} u-2 l v F+2 l(1+v) v v_{2}-8 v\left(v_{2}\right)^{2} \\
+2(m+l) v v_{2}+4 l v_{2}+2 \alpha(1+v) v^{\frac{3}{2}}-2(1+v)^{\frac{3}{2}} v_{2} e^{u} K\left(e^{u} \xi\right) \\
+2 v_{1} v_{2}-\frac{8\left(v_{2}\right)^{2}}{1+v}+2 A^{a b} \tilde{D}_{a}^{c} u \tilde{D}_{b c} u+4 E_{1}+E_{2}+2 E_{3}+2 E_{4} .
\end{gathered}
$$


Remarquons que l'égalité (4.17) implique aussi la suivante :

$$
\tilde{D}_{c}\left(v_{2}\right) \tilde{D}^{c} u=\mu_{a} \tilde{D}_{a} v \tilde{D}^{a} u+2 v_{2}\left(v-v_{2}\right)
$$

de sorte que le développement de $\tilde{D}_{c} F \tilde{D}^{c} u$ donne

$$
\begin{gathered}
2 \tilde{D}_{c} F \tilde{D}^{c} u=-4 v_{2}\left(v-v_{1}\right)-2 \mu_{a} \tilde{D}_{a} v \tilde{D}^{a} u+2(m-1) \tilde{D}_{a} v \tilde{D}^{a} u \\
-\sqrt{1+v} e^{u}\left[3 K\left(e^{u} \xi\right) \tilde{D}_{a} v \tilde{D}^{a} u+2(1+v)\left(\tilde{D}_{c} K\right)\left(e^{u} \xi\right) \tilde{D}^{c} u\right] \\
-2 v(1+v)^{\frac{3}{2}} e^{u} \frac{\partial[\rho K(\rho \xi)]}{\partial \rho}\left(e^{u} \xi\right) .
\end{gathered}
$$

Utilisons (4.5), on montre que

$$
\begin{gathered}
2 \tilde{D}_{c} F \tilde{D}^{c} u-2 l v F=l(1+v) F-l(m-1)(1+v)^{2}+l(1+v) v_{2} \\
-2(1+v)^{\frac{3}{2}} e^{u}\left[v \frac{\partial[\rho K(\rho \xi)]}{\partial \rho}\left(e^{u} \xi\right)+\left(\tilde{D}_{c} K\right)\left(e^{u} \xi\right) \tilde{D}^{c} u\right] \\
-4\left(v_{2}\right)^{2}-2 l v_{2}+l(1+v)^{\frac{3}{2}} e^{u} K\left(e^{u} \xi\right) .
\end{gathered}
$$

Reportons cette égalité dans (4.19), la relation qui en résulte se transforme comme suit :

$$
\begin{gathered}
A^{a b} \tilde{D}_{a b} v-\frac{A^{a b} \tilde{D}_{a} v \tilde{D}_{b} v}{(1+v)}-l(1+v) F \geq l(1+v)^{\frac{3}{2}} e^{u} K\left(e^{u} \xi\right)+2\left(v_{2}\right)^{2} \\
+\frac{8 v_{2}\left(1+v_{1}\right)}{1+v}+l(1+v) v_{2}+2(m+5) v v_{2}+8(1+v) v_{1} v_{2} \\
-l(m-1)(1+v)^{2}-2(1+v)^{\frac{3}{2}} e^{u}\left(\tilde{D}_{a} K\right)\left(e^{u} \xi\right) \tilde{D}^{a} u \\
+2 v(1+v)\left[\alpha \sqrt{v}-\sqrt{1+v} e^{u} \frac{\partial[\rho K(\rho \xi)]}{\partial \rho}\left(e^{u} \xi\right)\right] \\
+2(1+v)^{\frac{3}{2}} v_{2}\left[(l-4) \sqrt{1+v}-e^{u} K\left(e^{u} \xi\right)\right] \\
+2 A^{a b} \tilde{D}_{a}^{c} u \tilde{D}_{b c} u+4 E_{1}+E_{2}+2 E_{3}+2 E_{4} .
\end{gathered}
$$

Supposons que $l \geq l_{0}=4+r_{2} \underset{\Sigma_{r_{1}, r_{2}}}{\max } K$, tenons compte du choix (4.3) de $\alpha$ et minorons par 0 les termes positifs, l'égalité précédente permet d'écrire

$$
\begin{gathered}
A^{a b} \tilde{D}_{a b} v-\frac{A^{a b} \tilde{D}_{a} v \tilde{D}_{b} v}{(1+v)}-l(1+v) F \geq-l(m-1)(1+v)^{2}+2 A^{a b} \tilde{D}_{a}^{c} u \tilde{D}_{b c} u \\
-2(1+v)^{2} e^{u}\|\tilde{D} K\|_{\infty}+4 E_{1}+E_{2}+2 E_{3}+2 E_{4} .
\end{gathered}
$$

Or, $\left|E_{2}\right| \leq C_{1} v^{2}$, où $C_{1}$ est fonction de $\|\mathcal{T}\|_{\infty},\|\nabla \mathcal{T}\|_{\infty},\|\tilde{\mathcal{R}}\|_{\infty}$ et $\|u\|_{\infty}$ et puisque, d'après (4.13), on sait que $\left|E_{4}\right| \leq C_{1}^{\prime} v^{2}$ et compte tenu de (4.15), on a : $\left|E_{3}\right| \leq C_{2} l v^{\frac{3}{2}}$, où les 
constantes $C_{1}^{\prime}$ et $C_{2}$ ne sont fonction que de $\|\mathcal{T}\|_{\infty}$ et $\|u\|_{\infty}$, l'inégalité (4.20) implique l'existence d'une constante positive $C_{3}$ telle que

$$
\begin{gathered}
A^{a b} \tilde{D}_{a b} v-\frac{1}{(1+v)} A^{a b} \tilde{D}_{a} v \tilde{D}_{b} v+l(1+v) F \geq-2 C_{3} l(1+v)^{2} \\
+2 A^{a b} \tilde{D}_{a}^{c} u \tilde{D}_{b c} u+4 E_{1} .
\end{gathered}
$$

Reportons cette inégalité dans (4.6), on oboutit à la suivante :

$$
-C_{3} l(1+v)^{2}+A^{a b} \tilde{D}_{a}^{c} u \tilde{D}_{b c} u+2 E_{1} \leq 0 .
$$

Maintenant, on développe le carré suivant :

$$
\begin{gathered}
K=G^{a b} G^{c d}\left[\tilde{D}_{a d} u-\epsilon \tilde{D}_{a} u \tilde{D}^{e} u \tilde{D}_{e d} u-e^{\left(\mu_{a}+\mu_{e}\right) u} T_{a e}^{l} \tilde{D}^{e} u G_{l d}\right] \\
{\left[\tilde{D}_{b c} u-\epsilon \tilde{D}_{b} u \tilde{D}^{e} u \tilde{D}_{e c} u-e^{\left(\mu_{b}+\mu_{e}\right) u} T_{b e}^{l} \tilde{D}^{e} u G_{l c}\right]}
\end{gathered}
$$

avec $\epsilon=\frac{1}{v}$ pour voir que

$$
\begin{gathered}
(1+v) K=A^{a b} \tilde{D}_{a}^{c} u \tilde{D}_{b c} u+(1+v) e^{\left(\mu_{a}+\mu_{e}+\mu_{b}+\mu_{f}\right) u} G^{a b} G_{c d} T_{a e}^{c} \tilde{D}^{e} u T_{b f}^{d} \tilde{D}^{f} u \\
+2 E_{1}-v^{-1} \tilde{D}^{a} u \tilde{D}^{b} u \tilde{D}_{a c} u \tilde{D}_{b}^{c} u+2 v^{-1} e^{\left(\mu_{b}+\mu_{e}\right) u} T_{b e}^{d} \tilde{D}^{e} u \tilde{D}^{a} u \tilde{D}^{b} u \tilde{D}_{a d} u
\end{gathered}
$$

Il existe, alors, une constante positive $C_{4}$ fonction de $\|u\|_{\infty}$ et $\|\mathcal{T}\|_{\infty}$, telle que

$$
\begin{gathered}
A^{a b} \tilde{D}_{a}^{c} u \tilde{D}_{b c} u+2 E_{1} \geq-C_{4}(1+v)^{2}+v^{-1} \tilde{D}^{a} u \tilde{D}^{b} u \tilde{D}_{a c} u \tilde{D}_{b}^{c} u \\
-2 v^{-1} e^{\left(\mu_{b}+\mu_{c}\right) u} \tilde{D}^{a} u \tilde{D}^{b} u \tilde{D}_{a}^{d} u T_{b c}^{e} \tilde{D}_{e} u .
\end{gathered}
$$

Utilisons (4.12), on montre que

$$
\begin{gathered}
\tilde{D}^{a} u \tilde{D}^{b} u \tilde{D}_{a c} u \tilde{D}_{b}^{c} u=\tilde{D}^{a} u \tilde{D}^{b} u \tilde{D}_{c a} u \tilde{D}_{b}^{c} u-2 \tilde{D}^{a} u \tilde{D}^{b} u \tilde{D}_{a}^{c} u e^{\left(\mu_{b}+\mu_{c}\right) u} T_{b c}^{e} \tilde{D}_{e} u \\
+G^{c d} \tilde{D}^{a} u \tilde{D}^{b} u e^{\left(\mu_{a}+\mu_{b}+\mu_{c}+\mu_{d}\right) u} T_{a c}^{e} \tilde{D}_{e} u T_{b d}^{f} \tilde{D}_{f} u
\end{gathered}
$$

et, donc, d'après (4.9), on aura :

$$
\begin{gathered}
\tilde{D}^{a} u \tilde{D}^{b} u \tilde{D}_{a c} u \tilde{D}_{b}^{c} u=\frac{1}{4} \tilde{D}^{a} v \tilde{D}_{a} v-v_{2} \tilde{D}^{a} v \tilde{D}_{a} u+v\left(v_{2}\right)^{2} \\
-\left(\tilde{D}^{b} u \tilde{D}^{c} v-2 v_{2} \tilde{D}^{b} u \tilde{D}^{c} u\right) e^{\left(\mu_{b}+\mu_{c}\right) u} T_{b c}^{e} \tilde{D}_{e} u \\
+G^{c d} \tilde{D}^{a} u \tilde{D}^{b} u e^{\left(\mu_{a}+\mu_{b}+\mu_{c}+\mu_{d}\right) u} T_{a c}^{e} \tilde{D}_{e} u T_{b d}^{f} \tilde{D}_{f} u
\end{gathered}
$$

Ainsi, tenons compte de (4.5), il existe une constante positive $C_{5}$ fonction de $\|u\|_{\infty}$ et $\|\mathcal{T}\|_{\infty}$, telle que

$$
\tilde{D}^{a} u \tilde{D}^{b} u \tilde{D}_{a c} u \tilde{D}_{b}^{c} u \geq \frac{l^{2}}{4}(1+v)^{2} v-l(1+v) v v_{2}-C_{5} l(1+v) v_{2} \sqrt{v} .
$$


De même, on vérifie que

$$
\begin{gathered}
2 \tilde{D}^{a} u \tilde{D}^{b} u \tilde{D}_{a d} u T_{b e}^{d} \tilde{D}^{e} u=T_{b e}^{d} \tilde{D}^{e} u \tilde{D}^{b} u \tilde{D}_{d} v-2 v_{2} T_{b e}^{d} \tilde{D}^{b} u \tilde{D}^{e} u \tilde{D}_{d} u \\
-2 e^{\left(\mu_{a}+\mu_{d}\right) u} T_{b e}^{d} T_{a d}^{f} \tilde{D}^{a} u \tilde{D}^{b} u \tilde{D}^{e} u \tilde{D}_{f} u
\end{gathered}
$$

et donc (4.5) implique l'existence d'une constante positive $C_{6}$ fonction de $\|u\|_{\infty}$ et $\|\mathcal{T}\|_{\infty}$, telle que

$$
\left|2 e^{\left(\mu_{b}+\mu_{c}\right) u} \tilde{D}^{a} u \tilde{D}^{b} u \tilde{D}_{a}^{d} u T_{b c}^{e} \tilde{D}_{e} u\right| \leq C_{6} l(1+v) v_{2} \sqrt{v} .
$$

Reportons (4.23) et (4.24) dans (4.22), compte tenu du fait que $v_{2} \leq 1+v$, on en déduit que

$$
A^{a b} \tilde{D}_{a}^{c} u \tilde{D}_{b c} u+2 E_{1} \geq\left(4^{-1} l^{2}-l-C_{4}\right)(1+v)^{2}-l C_{7}(1+v)^{\frac{3}{2}},
$$

où $C_{7}=C_{5}+C_{6}$. Une inégalité qui, compte tenu de (4.21), implique

$$
\left(4^{-1} l^{2}-l C_{3}-l-C_{4}\right) \sqrt{1+v}-l C_{7} \leq 0 .
$$

De sorte que, pour $l$ assez grand, on aura : $1+v(\xi) \leq C_{8}=\left(l C_{7}\right)^{2}$. Ainsi, compte tenu de (4.18), $1+v \leq 1+C_{8}$. La définition (4.4) de la fonctionnelle $\Gamma$ montre que partout dans $\Sigma$, on a :

$$
v \leq\left(1+C_{8}\right)\left(\frac{r_{2}}{r_{1}}\right)^{l}
$$

Le lemme est prouvé.

Lemme 2. Soient $K \in \mathscr{C}^{\infty}\left(E_{*}\right)$ une fonction partout strictement positive vérifiant l'hypothèse (1.1) du théorème 2. Alors toute solution $u \in C^{3}(\Sigma)$ d'une équation de la forme

$$
\sum_{n+1 \leq \alpha, \beta \leq n+m-1} B^{\alpha \beta}(u) D_{\alpha \beta} u=(m-1)\left(1+v_{1}\right)-\left(1+v_{1}\right)^{\frac{3}{2}} e^{u} K\left(e^{u} \xi\right),
$$

où $B^{\alpha \beta}=\left(1+v_{1}\right) G^{\alpha \beta}-D^{\alpha} u D^{\beta} u$, vérifie $r_{1} \leq e^{u} \leq r_{2}$. Si en outre $K$ vérifie l'hypothèse de monotonicité (1.2) du théorème 2 , il existe alors une constante positive $C_{0}$ ne dépendant que de $n, r_{1}, r_{2}, \max _{\Sigma_{r_{1}, r_{2}}} K$ et $\|K\|_{\mathscr{C}^{1}\left(\Sigma_{r_{1}, r_{2}}\right)}$ telle que $v_{1} \leq C_{0}$.

Démonstration. Soit $u \in \mathscr{C}^{3}(\Sigma)$ une solution de (4.25). Soit $\xi \in \Sigma$ un point où $u$ atteint son maximum. Si $u(\xi)>\log \left(r_{2}\right)$, l'hypothèse de croissance (1.1) faite sur $K$ implique qu'au point $\xi$, on aura

$$
0 \geq \tilde{D}_{\alpha}^{\alpha} u=(m-1)-e^{u} K\left(e^{u} \xi\right)>0
$$

ce qui constitue une contradiction. La minoration $u \geq \log \left(r_{1}\right)$ s'obtient par analogie en considérant un point où $u$ atteint son minimum.

A présent, on dérive une fois l'équation (4.25) suivant une direction verticale, il vient

$$
B^{\alpha \beta} D_{\lambda \alpha \beta} u+D_{\lambda} B^{\alpha \beta} D_{\alpha \beta} u=D_{\lambda} F
$$


où $F$ désigne le second membre de (4.25). Développons $D_{\lambda} B^{\alpha \beta}$ et saturons par $D^{\lambda} u$, on obtient,

$$
B^{\alpha \beta} D_{\lambda \alpha \beta} u D^{\lambda} u+\triangle_{1} u D_{\lambda} v_{1} D^{\lambda} u-\frac{1}{2} D_{\lambda} v_{1} D^{\lambda} \tilde{v}=D_{\lambda} F D^{\lambda} u,
$$

où l'on a noté $\triangle_{1} u=G^{\alpha \beta} D_{\alpha \beta} u$. Ce terme peut être exprimé à partir de (4.25) de la manière suivante :

$$
G^{\alpha \beta} D_{\alpha \beta} u=\frac{F+D^{\alpha} u D^{\beta} u D_{\alpha \beta} u}{1+v_{1}}
$$

et puisque $D^{\beta} u D_{\alpha \beta} u=\frac{1}{2} D_{\alpha} v_{1}$, on obtient

$$
G^{\alpha \beta} D_{\alpha \beta} u=\frac{F}{1+v_{1}}+\frac{1}{2} \frac{D^{\alpha} u D_{\alpha} v_{1}}{1+v_{1}}
$$

Par suite

$$
\triangle_{1} u D_{\lambda} v_{1} D^{\lambda} u=\frac{F}{1+v_{1}} D_{\lambda} v_{1} D^{\lambda} u+\frac{1}{2} \frac{D^{\alpha} u D^{\lambda} u D_{\alpha} v_{1} D_{\lambda} v_{1}}{1+v_{1}}
$$

Mais, d'après l'expression des composantes $B^{\alpha \beta}$ qui implique que

$$
D^{\alpha} u D^{\beta} u=\left(1+v_{1}\right) G^{\alpha \beta}-B^{\alpha \beta},
$$

on peut écrire

$$
\triangle_{1} u D_{\lambda} v_{1} D^{\lambda} u=\frac{F}{1+v_{1}} D_{\lambda} v_{1} D^{\lambda} u+\frac{1}{2} D_{\lambda} v_{1} D^{\lambda} v_{1}-\frac{1}{2} \frac{B^{\alpha \beta} D_{\alpha} v_{1} D_{\beta} v_{1}}{1+v_{1}} .
$$

D'autre part, et puisque $D_{\lambda \alpha \beta} u=D_{\alpha \beta \lambda} u+\tilde{R}_{\beta \alpha \lambda}^{A} D_{A} u$, les expressions (2.15) et (2.16) donnant les composantes de la forme $\tilde{R}_{\beta \alpha \lambda}^{A}$ du tenseur de courbure, impliquent que

$$
B^{\alpha \beta} D_{\lambda \alpha \beta} u D^{\lambda} u=B^{\alpha \beta} D_{\alpha \beta \lambda} u D^{\lambda} u-(m-2) v_{1}\left(1+v_{1}\right) .
$$

Reportons (4.28) et (4.29) dans (4.26) et multiplions par 2, la relation qui en résulte s'écrit sous la forme :

$$
\begin{gathered}
2 B^{\alpha \beta} D_{\alpha \beta \lambda} u D^{\lambda} u-\frac{B^{\alpha \beta} D_{\alpha} v_{1} D_{\beta} v_{1}}{1+v_{1}}=2(m-2) v_{1}\left(1+v_{1}\right) \\
+2 D_{\lambda} F D^{\lambda} u-\frac{2 F D_{\lambda} v_{1} D^{\lambda} u}{1+v_{1}} .
\end{gathered}
$$

Maintenant, on considère la fonctionnelle

$$
\Gamma(u)=\left(1+v_{1}\right) e^{l u},
$$

où $l$ est un réel strictement positif fixé ultérieurement. En un point $\xi \in \Sigma$ où $\Gamma(u)$ atteint son maximum, on a :

$$
{ }_{f} r a c D_{\lambda} \Gamma \Gamma=\frac{D_{\lambda} v_{1}}{1+v_{1}}+l D_{\lambda} u=0
$$

et $B^{\alpha \beta} D_{\alpha \beta} \Gamma \leq 0$, c'est-à-dire, tenons compte du fait que $u$ vérifie l'équation (4.25),

$$
B^{\alpha \beta} D_{\alpha \beta} v_{1}-\frac{B^{\alpha \beta} D_{\alpha} v_{1} D_{\beta} v_{1}}{1+v_{1}}+l\left(1+v_{1}\right) F \leq 0 .
$$


Or,

$$
B^{\alpha \beta} D_{\alpha \beta} v_{1}=2 B^{\alpha \beta} D_{\alpha \beta \lambda} u D^{\lambda} u+2 B^{\alpha \beta} D_{\alpha \lambda} u D_{\beta}^{\lambda} u .
$$

Donc, d'après (4.30) et puisque $(m-2) v_{1}\left(1+v_{1}\right) \geq 0$, au maximum de $\Gamma$, on doit avoir

$$
2 B^{\alpha \beta} D_{\alpha \lambda} u D_{\beta}^{\lambda} u+l\left(1+v_{1}\right) F+2 D_{\lambda} F D^{\lambda} u-\frac{2 F D_{\lambda} v_{1} D^{\lambda} u}{1+v_{1}} \leq 0
$$

c'est-à-dire, tenons compte de (4.32),

$$
2 B^{\alpha \beta} D_{\alpha \lambda} u D_{\beta}^{\lambda} u+l F+3 l v_{1} F+2 D_{\lambda} F D^{\lambda} u \leq 0 .
$$

Développons $D_{\lambda} F D^{\lambda} u$. Compte tenu de (4.32), on vérifie que

$$
\begin{aligned}
& 2 D_{\lambda} F D^{\lambda} u+3 l v_{1} F=-2\left(1+v_{1}\right)^{\frac{3}{2}} e^{u}\left(D_{\lambda} K\right)\left(e^{u} \xi\right) D^{\lambda} u \\
& +l(m-1)\left(1+v_{1}\right) v_{1}-2 v_{1}\left(1+v_{1}\right)^{\frac{3}{2}} e^{u} \frac{\partial[\rho K(\rho \xi)]}{\partial \rho}\left(e^{u} \xi\right) .
\end{aligned}
$$

L'hypothèse de croissance (1.2) faite sur $K$ implique donc l'existence d'une constante positive $C_{1}$, fonction de $\|K\|_{\mathscr{C}^{1}\left(\Sigma_{r_{1}, r_{2}}\right)}$, telle que

$$
2 D_{\lambda} F D^{\lambda} u+3 l v_{1} F \geq\left(l-C_{1}\right)(m-1)\left(1+v_{1}\right) v_{1} .
$$

Reportons dans (4.33), on obtient

$$
2 B^{\alpha \beta} D_{\alpha \lambda} u D_{\beta}^{\lambda} u+l F+\left(l-C_{1}\right)(m-1)\left(1+v_{1}\right) v_{1} \leq 0
$$

et par suite, minorons par 0 les termes positifs, on aboutit à l'inégalité suivante :

$$
\left(l-C_{1}\right)(m-1)\left(1+v_{1}\right) v_{1}-l\left(1+v_{1}\right)^{\frac{3}{2}} e^{u} K\left(e^{u} \xi\right) \leq 0 .
$$

Posons $l=1+C_{1}$. Si

$$
v_{1}(\xi) \geq 1
$$

l'inégalité (4.34) combinée avec le fait que $m \geq 2$ montre que

$$
\sqrt{v_{1}} \leq 3 \sqrt{2}\left(1+C_{1}\right) e^{u} K\left(e^{u} \xi\right) \leq 3 \sqrt{2} r_{2}\left(1+C_{1}\right) K\left(e^{u} \xi\right)
$$

En conséquence, compte tenu de (4.35), on voit que

$$
v_{1}(\xi) \leq C_{2}=1+18\left[r_{2}\left(1+C_{1}\right) \max _{\Sigma_{r_{1}, r_{2}}} K\right]^{2} .
$$

Enfin, usons de la définition (4.31) de la fonctionnelle $\Gamma$, on vérifie que, partout dans $\Sigma$, on a :

$$
v_{1} \leq 1+v_{1} \leq C_{2}\left(\frac{r_{2}}{r_{1}}\right)^{l} .
$$

Le lemme est prouvé. 


\section{Preuve des résultats}

\subsection{Preuve du théorème 1}

Les calculs de la première section montrent que la courbure moyenne de $\Sigma_{r}$ est

$$
\mathcal{M}_{\Sigma_{r}}=-G^{a b} G\left(D_{e_{a}} e_{b}, \nu\right)=\frac{m-1}{r} .
$$

Définissons $\mathcal{Y}$ sur $\Sigma$ en posant

$$
\mathcal{Y}(\xi)=r \xi \text { pour tout } \xi \in \Sigma \text {. }
$$

Le graphe $\mathcal{Y}$ n'est autre que $\Sigma_{r}$ et s'il existe un réel $r>0$ tel que

$$
K(r \xi)=(m-1) r^{-1}, \text { quel que soit } \xi \in \Sigma,
$$

alors

$$
\mathcal{M}_{\mathcal{Y}}(r \xi)=K(r \xi), \text { pour tout } \xi \in \Sigma .
$$

Ceci montre la suffisance des hypothèses faites sur $K$ dans le théorème 1 .

Supposons qu'il existe un graphe radial $\mathcal{Y}$ sur $\Sigma$ de classe $\mathscr{C}^{\infty}$ dont la courbure moyenne est égale à $K$. Ceci est équivaut à dire qu'il existe une fonction $u \in C^{\infty}(\Sigma)$ vérifiant l'équation (3.4), mise en évidence à la troisième section. Notons

$$
r_{1}=\min _{\Sigma} e^{u} \text { et } r_{2}=\max _{\Sigma} e^{u} .
$$

On se place successivement en un point où $u$ atteint son minimum et en un point où $u$ atteint son maximum, on voit que

$$
(m-1)\left(r_{1}\right)^{-1} \geq K\left(r_{1}\right) \text { et }(m-1)\left(r_{2}\right)^{-1} \leq K\left(r_{2}\right) .
$$

Le résultat devient une conséquence de la continuité de $K$ via le théorème des valeurs intermédiaires.

\subsection{Preuve du théorème 2}

Pour tout entier naturel $k$ et tout réel $\alpha$ compris entre 0 et 1 , on notera par $A^{k, \alpha}(\Sigma)$ l'ensemble des fonctions $u \in \mathscr{C}^{k, \alpha}(\Sigma)$ dont la composante horizontale du gradient est identiquement nulle muni de la norme $\mathscr{C}^{k, \alpha}(\Sigma)$.

La preuve du théorème repose sur le lemme suivant.

Lemme 3. Soit $w \in A^{k+1, \alpha}(\Sigma)$. Pour tout $u \in \mathscr{C}^{k+2, \alpha}(\Sigma)$, on pose

$$
L[w](u)=\sum_{n+1 \leq \alpha, \beta \leq n+m-1} B^{\alpha \beta}(w) D_{\alpha \beta} u-u
$$

où $B^{\alpha \beta}(w)=\left(1+v_{1}(w)\right) G^{\alpha \beta}-D^{\alpha} w D^{\beta} w$ et $v_{1}(w)=D_{\alpha} w D^{\alpha} w$ est le carré de la norme de la composante verticale du gradient de $w$. L'opérateur $L[w]$ ainsi défini réalise un isomorphisme de $A^{k+2, \alpha}(\Sigma)$ sur $A^{k, \alpha}(\Sigma)$. 
Démonstration. Tout d'abord, on applique la méthode de continuité, combinée avecles inégalités de Schauder, pour montrer que pour toute fonction $w \in C^{k+1, \alpha}(\Sigma)$, l'opérateur elliptique $\tilde{L}[w]$ défini, pour $u \in C^{k+2, \alpha}(\Sigma)$, par

$$
\tilde{L}[w] u=G^{i j} D_{i j} u+B^{\alpha \beta}(w) D_{\alpha \beta} u-u
$$

est un isomorphisme de $C^{k+2, \alpha}(\Sigma)$ sur $C^{k, \alpha}(\Sigma)$. On en déduit que pour toute fonction $w \in A^{k+1, \alpha}(\Sigma)$, l'opérateur $L[w]$ est un isomorphisme de $A^{k+2, \alpha}(\Sigma)$ sur $A^{k, \alpha}(\Sigma)$. En effet, le principe du maximum implique que le noyau de celui-ci est trivial et pour toute fonction $z \in A^{k, \alpha}(\Sigma)$, il existe $u \in C^{k+2, \alpha}(\Sigma)$ telle que

$$
\tilde{L}[w] u=z,
$$

eu égard au fait que $\tilde{L}[w]$ est inversible. Or $z \in A^{k, \alpha}(\Sigma)$, donc $G^{k l} D_{k l} z=0$. Ainsi, dérivons deux fois (5.1) suivant des directions horizontales $k$ et $l$ et saturons par $G^{k l}$, compte tenu de l'expression (2.15) donnant les composantes de la courbure de $D$ et puisque les dérivées covariantes horizontales de $w$ sont identiquement nulles, on obtient

$$
G^{i j} D_{i j}\left(G^{k l} D_{k l} u\right)+B^{\alpha \beta}(w) D_{\alpha \beta}\left(G^{k l} D_{k l} u\right)-G^{k l} D_{k l} u=0
$$

ce qui signifie que $G^{k l} D_{k l} u$ est dans le noyau de $\tilde{L}[w]$, donc, ce terme est identiquement nul et par suite (5.1) se réduit à la forme suivante :

$$
L[w](u)=z .
$$

Dérivons l'équation (5.2) par rapport à une direction horizontale $i \in\{1, \ldots, n\}$, on obtient

$$
B^{\alpha \beta}(w) D_{i \alpha \beta} u+D_{i} B^{\alpha \beta}(w) D_{\alpha \beta} u-D_{i} u=0 .
$$

Saturons par $D^{i} u$, on voit que,

$$
B^{\alpha \beta} D_{i \alpha \beta} u D^{i} u+D_{i} B^{\alpha \beta}(w) D_{\alpha \beta} u D^{i} u-D_{i} u D^{i} u=0 .
$$

Tenons compte du fait que $w \in A^{k+1, \alpha}(\Sigma)$, qui implique que

$$
D_{i} B^{\alpha \beta}(w) D_{\alpha \beta} u D^{i} u=0,
$$

on aboutit à l'égalité suivante

$$
B^{\alpha \beta} D_{i \alpha \beta} u D^{i} u-\tilde{v}_{2}=0
$$

où $\tilde{v}_{2}=D_{i} u D^{i} u$ désigne le carré de la norme de la composante horizontale du gradient de $u$. D'après (2.15), on a $D_{i \alpha \beta} u=D_{\alpha \beta i} u$ et donc l'égalité (5.3) s'écrit

$$
B^{\alpha \beta} D_{\alpha \beta i} u D^{i} u-\tilde{v}_{2}=0
$$

D'autre part, en un point $\xi_{0}$ où $\tilde{v}_{2}$ atteint son maximum, on a : $B^{\alpha \beta} D_{\alpha \beta} \tilde{v}_{2} \leq 0$. Une inégalité que se traduit par

$$
B^{\alpha \beta} D_{\alpha \beta i} u D^{i} u+B^{\alpha \beta} D_{\alpha i} u D_{\beta}^{i} u \leq 0
$$

ce qui, compte tenu de (5.4) et puisque le terme $B^{\alpha \beta} D_{\alpha i} u D_{\beta}^{i} u$ est positif, implique que $\tilde{v}_{2}\left(\xi_{0}\right)=0$. Ainsi $\tilde{v}_{2}$ est partout nul et le lemme est prouvé. 
A présent, pour tout $t \in[0,1]$ et pour tout $w \in A^{1, \alpha}(\Sigma)$, on pose $T_{t} w=u_{t}$, où $u_{t} \in A^{2, \alpha}(\Sigma)$ est l'unique solution de

$$
L[w] u_{t}=t\left[-w+(m-1)\left[1+v_{1}(w)\right]-\left[1+v_{1}(w)\right]^{\frac{3}{2}} e^{w} K\left(e^{w} \xi\right)\right] .
$$

Le lemme 3 montre que $u_{t} \in A^{2, \alpha}(\Sigma)$ et la théorie elliptique implique que

$$
\left\|u_{t}\right\|_{\mathscr{C}^{2, \alpha}(\Sigma)} \leq \text { Cste }
$$

La famille d'opérateurs $T_{t}$ vérifie les propriétés suivantes :

(i) Pour tout $w \in A^{1, \alpha}(\Sigma), T_{0} w=0$.

(ii) A $t$ fixé, $w \mapsto T_{t} w$ est un opérateur compact de $A^{1, \alpha}(\Sigma)$ dans $A^{1, \alpha}(\Sigma)$. En effet, soit $\Gamma \subset A^{1, \alpha}(\Sigma)$ qu'on suppose borné dans $A^{1, \alpha}(\Sigma)$. Donc, d'après (5.6), $\left\{T_{t} w \mid\right.$ $w \in \Gamma\}$ est un sous ensemble de $A^{2, \alpha}(\Sigma)$ qui est uniformément borné dans $C^{2, \alpha}(\Sigma)$. L'ensemble $C^{2, \alpha}(\Sigma)$ étant un compact de $C^{1, \alpha}(\Sigma)$, on en déduit que $\left\{T_{t} w \mid w \in \Gamma\right\}$ est un sous ensemble de $A^{1, \alpha}(\Sigma)$ relativement compact.

(iii) A $w$ fixé, $t \mapsto T_{t} w$ est continue, sinon, il existerait une suite $\left(t_{i}\right)$ dans l'intervalle $[0,1]$ et un réel $\epsilon>0$ tels que $\lim _{i \rightarrow \infty} t_{i}=t$ et, pour $i$ assez grand,

$$
\left\|\tilde{u}_{t_{i}}-\tilde{u}_{t}\right\|_{C^{1, \alpha}(\Sigma)}>\epsilon .
$$

L'estimée (5.6) implique que la suite $\left(\tilde{u}_{t_{i}}\right)$ est bornée dans $C^{2, \alpha}(\Sigma)$. L'injection de $C^{2, \alpha}(\Sigma)$ dans $C^{1, \alpha}(\Sigma)$ étant compacte, donc quitte à en extraire une sous-suite, on peut supposer que $\left(\tilde{u}_{t_{i}}\right)$ converge dans $C^{1, \alpha}(\Sigma)$. D'après $(5.5)$, et puisque $\tilde{u}_{t_{i}}$ est de classe $C^{2, \alpha}(\Sigma)$, sa limite est $\tilde{u}_{t}$. Il en découle que, pour $i$ assez grand,

$$
\left\|\tilde{u}_{t_{i}}-\tilde{u}_{t}\right\|_{C^{1, \alpha}(\Sigma)}<\epsilon / 2
$$

Ceci contredit (5.7).

Compte tenu des propriétés (i), (ii) et (iii), l'opérateur $T$ défini en posant

$$
T(t, w)=w-T_{t} w \text { pour }(t, w) \in[0,1] \times A^{1, \alpha}(\Sigma)
$$

est une déformation compacte de l'identité de $A^{1, \alpha}(\Sigma)$. Notre but est de résoudre l'équation

$$
T(t, u)=0, \text { pour } t \in[0,1]
$$

dans $A^{1, \alpha}(\Sigma)$. Le lemme 2 et la théorie classique des équations elliptiques, cf. [6], implique qu'une telle solution $u$ est dans $A^{2, \alpha}(\Sigma)$ et qu'il existe un réel $R>0$ telle que

$$
\|u\|_{\mathscr{C}^{1, \alpha}(\Sigma)}<R
$$

On note alors

$$
B_{R}=\left\{u \in A^{1, \alpha}(\Sigma) m i d\|u\|_{\mathscr{C}^{1, \alpha}(\Sigma)}<R\right\} .
$$

Compte tenu de (5.9), l'équation (5.8) n'admet pas de solution $u$ sur le bord $\partial B_{R}$ de $B_{R}$. La déformation $T$ est donc une homotopie compacte sur le bord de $B_{R}$. En conséquence, 
d'après [3], (théorème 5.3.14), le dégré de Leray-Schauder de $T$ en 0 relativement à $B_{R}$ ne dépend pas de $t$. Ainsi, pout tout $t \in[0,1]$, on a :

$$
d\left(T(t, .), 0, B_{R}\right)=d\left(T(0, .), 0, B_{R}\right)=d\left(I d, 0, B_{R}\right)=1 .
$$

En particulier, l'opérateur $T_{1}$ admet un point fixe $u \in A^{1, \alpha}(\Sigma)$, solution de classe $A^{2, \alpha}(\Sigma)$ de l'équation

$$
\sum_{n+1 \leq \alpha, \beta \leq n+m-1} B^{\alpha \beta} D_{\alpha \beta} u=(m-1)\left(1+v_{1}\right)-\left(1+v_{1}\right)^{\frac{3}{2}} e^{u} K\left(e^{u} \xi\right) .
$$

Or $v_{2}(u)$ est partout nul, notre solution vérifie en fait l'équation

$$
A^{a b} \tilde{D}_{a b} u=-v_{2}+(m-1)(1+v)-(1+v)^{\frac{3}{2}} e^{u} K\left(e^{u} \xi\right) .
$$

Une équation qui est uniformément elliptique ce qui permet de conclure en ce qui concerne la régularité $\mathscr{C}^{\infty}(\Sigma)$ de toute solution de classe $\mathscr{C}^{2, \alpha}(\Sigma)$.

\subsection{Preuve du théorème 3}

La fonction $K \in \mathscr{C}^{\infty}\left(E_{*}\right)$ est donnée telle qu'il existe deux réels $r_{1}$ et $r_{2}$ tels que $0<r_{1} \leq 1 \leq r_{2}$ et

$$
K(\xi)>\frac{m-1}{\|\xi\|} \text { si }\|\xi\|<r_{1}, K(\xi)<\frac{m-1}{\|\xi\|} \text { si }\|\xi\|>r_{2} .
$$

On cherche à résoudre dans $\Sigma$ l'équation elliptique suivante :

$$
A^{a b}(u) \tilde{D}_{a b} u=-v_{2}+(m-1)(1+v)-(1+v)^{\frac{3}{2}} e^{u} K\left(e^{u} \xi\right)
$$

Tout d'abord, on note $\Sigma^{\prime}=\left\{\xi \in E \mid r_{1} \leq\|\xi\| \leq r_{2}\right\}, \nu$ le champ radial unitaire et $r$ la fonction $r(\xi)=\|\xi\|$. Soit $w \in \mathscr{C}^{\infty}\left(\Sigma^{\prime}\right)$, on désigne par $w_{1}$ la restriction de $w$ à $\Sigma$ que l'on prolonge en une fonction radialement constante et pour $u \in \mathscr{C}^{\infty}\left(\Sigma^{\prime}\right)$, on note $\tilde{D}^{a} u=e^{\mu_{a} w_{1}} D^{a} u, \tilde{D}^{a b} u=e^{\left(\mu_{a}+\mu_{b}\right) w_{1}} D^{a b} u$,

$$
\begin{aligned}
& v_{1}(u)=\left(1-\mu_{a}\right) \tilde{D}_{a} u \tilde{D}^{a} u, v_{2}(u)=\mu_{a} \tilde{D}_{a} u \tilde{D}^{a} u, \tilde{v}(u)=r^{2} v_{1}(u)+v_{2}(u), \\
& F(w)=-v_{2}\left(w_{1}\right)+(m-1)\left[1+\tilde{v}\left(w_{1}\right)\right]-\left[1+\tilde{v}\left(w_{1}\right)\right]^{3 / 2} e^{w_{1}} K\left(e^{w_{1}} \frac{\xi}{\|\xi\|}\right), \\
& A^{a b}(w)=\left(1+\tilde{v}\left(w_{1}\right)\right) G^{a b}-r^{2-\mu_{a}-\mu_{b}} \tilde{D}^{a} w_{1} \tilde{D}^{b} w_{1},
\end{aligned}
$$

et $B(w)=(m-1)\left[1+\tilde{v}\left(w_{1}\right)\right]-r^{2} v_{1}\left(w_{1}\right)$. Si $t \in[0,1]$ et $w \in \mathscr{C}^{\infty}\left(\Sigma^{\prime}\right)$, on désigne par $\tilde{u}_{t}$ l'unique solution du problème de Neumann

$$
\left\{\begin{array}{c}
D_{\nu \nu} u+A^{a b}(w) r^{2-\mu_{a}-\mu_{b}} \tilde{D}_{a b} u+\mu_{a} \log (r) \tilde{D}_{a}^{a} u-u=t\left[-w_{1}+F(w)\right] \\
+D_{\nu \nu} w+r B(w) D_{\nu} w-t \alpha \log (r) \sqrt{\tilde{v}\left(w_{1}\right)} \text { dans } \Sigma^{\prime} \\
D_{\nu} u=0 \text { sur } \partial \Sigma^{\prime},
\end{array}\right.
$$


où

$$
\alpha=2 \sup _{\xi \in \Sigma} \sup _{\Sigma^{\prime}}\left|r \frac{\partial[r K(r \xi)]}{\partial r}\right| .
$$

La preuve de l'existence d'une solution $\tilde{u}_{t} \in C^{2, \alpha}\left(\Sigma^{\prime}\right)$ de (5.12) ainsi que de l'existence d'une constante positive $C$ telle que

$$
\left\|\tilde{u}_{t}\right\|_{C^{2, \alpha}\left(\Sigma^{\prime}\right)} \leq C
$$

est très classique. On renvoie à [1] et [4], voir aussi [5], quant à l'unicité, elle découle du principe du maximum. L'équation (5.12) étant elliptique et toutes les données sont de classe $\mathscr{C}^{\infty}$, la solution $\tilde{u}_{t}$ est de classe $C^{\infty}\left(\Sigma^{\prime}\right)$ par régularité. En particulier, étant donnée une partie $\mathcal{B}$ bornée de $\mathscr{C}^{\infty}\left(\Sigma^{\prime}\right)$, il existe $\tilde{u}_{t} \in \mathscr{C}^{\infty}\left(\Sigma^{\prime}\right)$ ainsi qu'une suite de réels positifs $\left(C_{k}\right)_{k \geq 0}$ telle que, quel que soit $(t, w) \in[0,1] \times \mathcal{B}$ et pour tout entier $k \geq 0$, on ait:

$$
\left\|\tilde{u}_{t}\right\|_{\mathscr{C}^{k}\left(\Sigma^{\prime}\right)} \leq C_{k}
$$

Il en découle que l'opérateur $T_{t}$ défini sur $\mathscr{C}^{\infty}\left(\Sigma^{\prime}\right)$ par $T_{t} w=\tilde{u}_{t}$ est compact. Il en est de même de l'opérateur $T$ défini de $[0,1] \times \mathscr{C}^{\infty}\left(\Sigma^{\prime}\right)$ vers $\mathscr{C}^{\infty}\left(\Sigma^{\prime}\right)$ par

$$
T(t, w)=w-T_{t} w .
$$

Notre objectif est de montrer que l'équation

$$
T(t, u)=0, \text { pour } t \in[0,1],
$$

admet une solution dans $\mathscr{C}^{\infty}\left(\Sigma^{\prime}\right)$. Si une telle solution existe, celle-ci, notée $\tilde{u}_{t}$, est une constante radiale. En effet, $\tilde{u}_{t}$ vérifie le système suivant :

$$
\left\{\begin{array}{c}
A^{a b}\left(\tilde{u}_{t}\right) r^{2-\mu_{a}-\mu_{b}} \tilde{D}_{a b} \tilde{u}_{t}+\mu_{a} \log (r) \tilde{D}_{a}^{a} \tilde{u}_{t}-\tilde{u}_{t}=t\left[-u_{t}+F\left(\tilde{u}_{t}\right)\right] \\
+r B\left(\tilde{u}_{t}\right) D_{\nu} \tilde{u}_{t}-t \alpha \log (r) \sqrt{\tilde{v}\left(u_{t}\right)} \text { dans } \Sigma^{\prime} \\
D_{\nu} \tilde{u}_{t}=0 \text { sur } \partial \Sigma^{\prime},
\end{array}\right.
$$

où l'on a noté par $u_{t}$ le prolongement en une constante radiale de la restriction à $\Sigma$ de $\tilde{u}_{t}$.

Dérivons radialement l'équation (5.16) et multiplions par $r$ l'équation ainsi obtenue. Du fait que $D_{\nu} r=1$ et puisque $u_{t}$ est une constante radiale, il en découle que

$$
\begin{gathered}
r D_{\nu}\left[A^{a b}\left(\tilde{u}_{t}\right)\right] r^{2-\mu_{a}-\mu_{b}} \tilde{D}_{a b} \tilde{u}_{t}+\left(2-\mu_{a}-\mu_{b}\right) r^{2-\mu_{a}-\mu_{b}} A^{a b}\left(\tilde{u}_{t}\right) \tilde{D}_{a b} \tilde{u}_{t} \\
+A^{a b}\left(\tilde{u}_{t}\right) r^{2-\mu_{a}-\mu_{b}} r \tilde{D}_{\nu a b} \tilde{u}_{t}+\mu_{a} \tilde{D}_{a}^{a} \tilde{u}_{t}+\mu_{a} \log (r) G^{a b} r \tilde{D}_{\nu a b} \tilde{u}_{t}-r D_{\nu} \tilde{u}_{t} \\
=\operatorname{tr} D_{\nu} F\left(\tilde{u}_{t}\right)+r B\left(\tilde{u}_{t}\right) D_{\nu} \tilde{u}_{t}+r D_{\nu} \tilde{u}_{t} r D_{\nu}\left[B\left(\tilde{u}_{t}\right)\right]+B\left(\tilde{u}_{t}\right) r^{2} D_{\nu \nu} \tilde{u}_{t} \\
-t \alpha \sqrt{\tilde{v}\left(u_{t}\right)}-t \alpha \log (r) r D_{\nu} \sqrt{r^{2} v_{1}\left(u_{t}\right)+v_{2}\left(u_{t}\right)} .
\end{gathered}
$$

Remarquons d'abord que par un calcul analogue à celui de la seconde section, on montre que

$$
D_{e_{a}}(r \nu)=\left(1-\mu_{a}\right) e_{a}
$$


où $\mu_{a}$ vaut 1 ou 0 selon que la direction $e_{a}$ est horizontale ou verticale. Ainsi, usons de la définition de la dérivée covariante, on peut écrire :

$$
D_{e_{a}}\left(r D_{\nu} u\right)=D^{2} u\left(e_{a}, r \nu\right)+D u\left(D_{e_{a}}(r \nu)\right)=r D_{\nu}\left(D_{a} u\right)+\left(1-\mu_{a}\right) D_{a} u .
$$

On en déduit que

$$
r D_{\nu}\left(D_{a} u\right)=D_{e_{a}}\left(r D_{\nu} u\right)-\left(1-\mu_{a}\right) D_{a} u
$$

et, en particulier, pour une constante radiale $u$, on a :

$$
\begin{gathered}
r D_{\nu} v_{1}(u)=2\left(1-\mu_{a}\right) D_{a}\left(r D_{\nu} u\right) D^{a} u-2 v_{1}(u)=-2 v_{1}(u) \\
r D_{\nu} v_{2}(u)=2 \mu_{a} \tilde{D}_{a}\left(r D_{\nu} u\right) \tilde{D}^{a} u+2 v_{2}(u) r D_{\nu} u=0
\end{gathered}
$$

Combinons cette relation avec le fait que $u_{t}$ est une constante radiale, on obtient :

$$
r D_{\nu}\left[r^{2} v_{1}\left(u_{t}\right)+v_{2}\left(u_{t}\right)\right]=0 .
$$

Ainsi, l'équation (5.17) se réduit à la suivante :

$$
\begin{gathered}
\left(2-\mu_{a}-\mu_{b}\right) r^{2-\mu_{a}-\mu_{b}} A^{a b}\left(\tilde{u}_{t}\right) \tilde{D}_{a b} \tilde{u}_{t}+A^{a b}\left(\tilde{u}_{t}\right) r^{2-\mu_{a}-\mu_{b}} r \tilde{D}_{\nu a b} \tilde{u}_{t} \\
+\mu_{a} \tilde{D}_{a}^{a} \tilde{u}_{t}+\mu_{a} \log (r) G^{a b} r \tilde{D}_{\nu a b} \tilde{u}_{t}-r D_{\nu} \tilde{u}_{t}=r B\left(\tilde{u}_{t}\right) D_{\nu} \tilde{u}_{t} \\
+B\left(\tilde{u}_{t}\right) r^{2} D_{\nu \nu} \tilde{u}_{t}-t \alpha \sqrt{r^{2} v_{1}\left(u_{t}\right)+v_{2}\left(u_{t}\right)} .
\end{gathered}
$$

D'autre part, la définition de la dérivée covariante nous permet d'écrire que

$$
D_{a b}\left(r D_{\nu} u\right)=D^{2}\left(r D_{\nu} u\right)\left(e_{a}, e_{b}\right)=e_{a}\left[D\left(r D_{\nu} u\right)\left(e_{b}\right)\right]-D\left(r D_{\nu} u\right)\left(D_{e_{a}} e_{b}\right) .
$$

D'où

$$
\begin{gathered}
D_{a b}\left(r D_{\nu} u\right)=e_{a}\left[D_{e_{b}}(D u(r \nu))\right]-\left(D_{e_{a}} e_{b}\right) D u(r \nu) \\
=D_{e_{a}}\left(D^{2} u\left(e_{b}, r \nu\right)+D_{e_{a}} D u\left(D_{e_{b}}(r \nu)\right)\right. \\
-D^{2} u\left(D_{e_{a}} e_{b}, r \nu\right)-D u\left(D_{D_{e_{a}} e_{b}}(r \nu)\right)
\end{gathered}
$$

et par suite

$$
\begin{aligned}
D_{a b}\left(r D_{\nu} u\right)= & D^{3} u\left(e_{a}, e_{b}, r \nu\right)+D^{2} u\left(e_{b}, D_{e_{a}}(r \nu)\right)+D^{2} u\left(e_{a}, D_{e_{b}}(r \nu)\right) \\
& +D u\left(D_{e_{a}}\left(D_{e_{b}}(r \nu)\right)\right)-D u\left(D_{D_{e_{a}} e_{b}}(r \nu)\right) .
\end{aligned}
$$

Or, la relation (5.18) permet d'écrire que

$$
D u\left(D_{e_{a}}\left(D_{e_{b}}(r \nu)\right)\right)=\left(1-\mu_{b}\right) D u\left(D_{e_{a}} e_{b}\right)
$$

et

$$
D u\left(D_{D_{e_{a}} e_{b}}(r \nu)\right)=\left(1-\mu_{b}\right) D u\left(D_{e_{a}} e_{b}\right) .
$$

Ainsi, usons à nouveau de (5.18), on obtient :

$$
D_{a b}\left(r D_{\nu} u\right)=D^{3} u\left(e_{a}, e_{b}, r \nu\right)+\left(2-\mu_{a}-\mu_{b}\right) D^{2} u\left(e_{a}, e_{b}\right)
$$


et d'après la définition de la connexion $D$ et celle de la dérivée covariante, on voit que

$$
D_{a b}\left(r D_{\nu} u\right)=r D_{\nu}\left(D_{a b} u\right)+\left(2-\mu_{a}-\mu_{b}\right) D_{a b} u .
$$

Eu égard à la relation (2.6) de la secande section donnant l'expression de la courbure de $D$ et dont découle l'égalité $D_{a b \nu} u=D_{\nu a b} u$. Reportons (5.21) dans (5.20), on obtient :

$$
\begin{gathered}
A^{a b}\left(\tilde{u}_{t}\right) r^{2-\mu_{a}-\mu_{b}} \tilde{D}_{a b}\left(r D_{\nu} \tilde{u}_{t}\right)+\mu_{a} \tilde{D}_{a}^{a} \tilde{u}_{t}+\mu_{a} \log (r) \tilde{D}_{a}^{a}\left(r D_{\nu} \tilde{u}_{t}\right) \\
-r D_{\nu} \tilde{u}_{t}=r B\left(\tilde{u}_{t}\right) D_{\nu} \tilde{u}_{t}+B\left(\tilde{u}_{t}\right) r^{2} D_{\nu \nu} \tilde{u}_{t}-t \alpha \sqrt{\tilde{v}\left(u_{t}\right)} .
\end{gathered}
$$

Rappellons que l'équation de Gauss et les calculs de la seconde section impliquent que pour deux fonctions $u_{1}, u_{2} \in \mathscr{C}^{2}\left(\Sigma^{\prime}\right)$ ayant les mêmes valeurs sur $\Sigma$, on a :

$$
D_{a b} u_{1}=D_{a b} u_{2}+\left(1-\mu_{a}\right) G_{a b} D_{\nu}\left(u_{1}-u_{2}\right), a, b \leq n+m-1 .
$$

Or $u_{t}=\left(\tilde{u}_{t}\right)_{\mid \Sigma}$. Donc, tenons compte de (5.23) et du fait que $D_{\nu} \tilde{u}_{t}$ est nul sur $\Sigma,(5.22)$ implique la relation suivante :

$$
\mu_{a} \tilde{D}_{a}^{a} u_{t}=-t \alpha \sqrt{v_{1}\left(u_{t}\right)+v_{2}\left(u_{t}\right)}, \text { partout dans } \Sigma \text {. }
$$

D'autre part, restreignons (5.16) à $\Sigma$, l'usage de (5.23) montre que la fonction $u_{t}$ vérifie l'équation suivante :

$$
A^{a b}\left(u_{t}\right) \tilde{D}_{a b} u_{t}-u_{t}=t\left[-u_{t}+F\left(u_{t}\right)\right], \text { partout dans } \Sigma .
$$

La fonction $u_{t}$ étant une constante radiale, une combinaison des équations (5.24) et (5.25), montre que $u_{t}$ est une autre solution de (5.16). En conséquence, on voit que

$$
\left\{\begin{array}{c}
A^{a b}\left(\tilde{u}_{t}\right) r^{2-\mu_{a}-\mu_{b}} \tilde{D}_{a b}\left(\tilde{u}_{t}-u_{t}\right)+\mu_{a} \log (r) \tilde{D}_{a}^{a}\left(\tilde{u}_{t}-u_{t}\right) \\
-\left(\tilde{u}_{t}-u_{t}\right)=r B\left(\tilde{u}_{t}\right) D_{\nu}\left(\tilde{u}_{t}-u_{t}\right) \text { dans } \Sigma^{\prime} \\
D_{\nu}\left(\tilde{u}_{t}-u_{t}\right)=0 \text { sur } \partial \Sigma^{\prime} .
\end{array}\right.
$$

Le principe du maximum montre que $\tilde{u}_{t}=u_{t}$ partout dans $\Sigma^{\prime}$. Ceci montre que $\tilde{u}_{t}$ est une constante radiale.

A présent, on montre que $u_{t}$ est estimée à priori $\mathscr{C}^{0}(\Sigma)$. Une majoration a priori se déduit immédiatement du principe du maximum. Soit $\xi \in \Sigma$ un point où $u_{t}$ atteint son maximum. Si $u_{t}(\xi)>\log \left(r_{2}\right)$, l'hypothèse de croissance (5.10) faite sur $K$ combinée avec l'équation (5.25) implique qu'au point $\xi$, on aura

$$
0 \geq \tilde{D}_{a}^{a} u_{t}=(1-t) u_{t}+t\left[(m-1)-e^{u_{t}} K\left(e^{u_{t}} \xi\right)\right]>0
$$

ce qui constitue une contradiction, eu égard au fait que $\log \left(r_{2}\right) \geq 0$. La minoration $u_{t} \geq \log \left(r_{1}\right)$ s'obtient par analogie en considérant un point où $u_{t}$ atteint son minimum.

La fonction $u_{t}$ vérifiant les équations (5.24) et (5.25), d'après le lemme 1 elle est estimée a priori dans $\mathscr{C}^{1}(\Sigma)$. La théorie classique des équations uniformément elliptiques, voir à titre d'exemple [1], [4], [6], montre comment obtenir l'estimée $\mathscr{C}^{1, \alpha}$. Plus précisément, 
on peut, par simple modification de la preuve du théorème 13.6 page 328 de Gilbarg et Trüdinger [4], conclure à l'existence d'un réel $c_{1}>0$ tel que

$$
\left\|u_{t}\right\|_{\mathscr{C}^{1, \alpha}(\Sigma)}<c_{1}
$$

La fonction $u_{t}$ étant une solution de l'équation (5.25), les inégalités de Schauder impliquent l'existence d'une constante positive $C$ telle que

$$
\left\|u_{t}\right\|_{\mathscr{C}^{2, \alpha}(\Sigma)}<C\left[\left\|u_{t}\right\|_{\mathscr{C}^{0}(\Sigma)}+\|F\|_{\mathscr{C}^{0, \alpha}\left(\Sigma^{\prime}\right)}\right]
$$

et donc il existe une constante positive $c_{2}$ telle que

$$
\left\|u_{t}\right\|_{\mathscr{C}^{2, \alpha}(\Sigma)}<c_{2}
$$

Supposons donc que que pour tout $s \leq k$, pour un $k \geq 2$, on ait :

$$
\left\|u_{t}\right\|_{\mathscr{C}^{s, \alpha}(\Sigma)}<c_{s}
$$

L'équation obtenue en dérivant $(k-1)$ fois l'équation (5.25) s'écrit localement sous la forme

$$
A^{a b} \tilde{D}_{a b}\left(D_{i_{1} i_{2} \ldots i_{k-1}} u_{t}\right)=H_{i_{1} i_{2} \ldots i_{k-1}}
$$

où le second membre $H_{i_{1} i_{2} \ldots i_{k-1}}$ ne dépend que des dérivées covariantes de $u_{t}$ d'ordre $\leq k$. Il est donc borné dans $\mathscr{C}^{0, \alpha}\left(\Sigma^{\prime}\right)$ et il en découle, d'après les inégalités de Schauder, que $\left\|D^{(k-1)} u_{t}\right\|_{\mathscr{C}^{2, \alpha}(\Sigma)}<c_{k+1}$, et par suite

$$
\left\|u_{t}\right\|_{\mathscr{C}^{k+1, \alpha}(\Sigma)}<c_{k+1}
$$

On vient ainsi d'établir par récurrence l'existence de réels positifs $a_{k}$ tels que

$$
\left\|u_{t}\right\|_{\mathscr{C}^{k}\left(\Sigma^{\prime}\right)}<a_{k}
$$

Eu égard au fait que $u_{t}$ est radialement constante. On note $B$ la pseudo-boule définie par

$$
B=\left\{u \in \mathscr{C}^{\infty}\left(\Sigma^{\prime}\right) \mid\|u\|_{\mathscr{C}^{k}\left(\Sigma^{\prime}\right)}<a_{k}\right\}
$$

Compte tenu de (5.26), l'équation (5.15) n'admet pas de solution sur le bord de $B$. La déformation $T$ est donc une homotopie compacte sur le bord de $B_{R}$. En conséquence, d'après le théorème de Nagumo [7], le dégré de $T$ en 0 relativement à $B$ ne dépend pas de $t$. Ainsi, pout tout $t \in[0,1]$, on :

$$
d(T(t, .), 0, B)=d(T(0, .), 0, B)=\gamma
$$

Or, pour $t=0$, la fonction $u_{0}=0$ est l'unique solution de (5.15) et l'on montre aisément que pour $w \in \mathscr{C}^{\infty}\left(\Sigma^{\prime}\right),\left(d_{u_{0}} T_{0}\right)(w)=u$, où $u$ est l'unique solution du problème suivant :

$$
\left\{\begin{array}{c}
D_{\nu \nu} u+r^{2-2 \mu_{a}} D_{a}^{a} u+\mu_{a} \log (r) D_{a}^{a} u-u=D_{\nu \nu} w \\
+(m-1) r D_{\nu} w \text { dans } \Sigma^{\prime} \\
D_{\nu} u=0 \text { sur } \partial \Sigma^{\prime}
\end{array}\right.
$$


A présent, si $w \in \operatorname{ker}\left[i d-\left(d_{u_{0}} T_{0}\right)\right]$, celle-ci sera solution du problème suivant :

$$
\left\{\begin{array}{c}
r^{2-2 \mu_{a}} D_{a}^{a} w+\mu_{a} \log (r) D_{a}^{a} w-w=(m-1) r D_{\nu} w \text { dans } \Sigma^{\prime} \\
D_{\nu} w=0 \text { sur } \partial \Sigma^{\prime}
\end{array}\right.
$$

Un raisonnement analogue à celui qui précède montre que $w$ est une constante radiale. Celle-ci étant identiquement nulle sur $\Sigma$, donc partout nulle et par suite

$$
\operatorname{ker}\left[\mathrm{id}-\left(d_{u_{0}} T_{0}\right)\right]=\{0\}
$$

Or, d'après sa définition et en raisonnant comme auparavant, on vérifie que $d_{u_{0}} T_{0}$ est un opérateur compact de $\mathscr{C}^{\infty}\left(\Sigma^{\prime}\right)$ vers $B^{1, \beta}\left(\Sigma^{\prime}\right), 0<\beta<\alpha$, et donc, tenons compte de (5.29), le théorème d'alternative de Fredholm implique que l'opérateur id $-\left(d_{u_{0}} T_{0}\right)$ est inversible. En conséquence 0 est un point régulier pour id $-T_{0}$. Ainsi, dans (5.27), $\gamma= \pm 1$ et en particulier, l'opérateur $T_{1}$ admet un point fixe de classe $\mathscr{C}^{\infty}\left(\Sigma^{\prime}\right)$. Celle-ci est une constante radiale qui est une solution de (5.11) dans $\Sigma$. Ceci achève la preuve du théorème.

\subsection{Remarques}

1- Expliquons ici en quel sens l'hypothèse de croissance des théorèmes 2 et 3 est la meilleure possible. Soit $K \in \mathscr{C}^{\infty}\left(E_{*}\right)$ une fonction strictement positive. On suppose qu'il existe un réel $a \in] 0,1[$ tel que

$$
K(\xi) \leq \frac{a(m-1)}{\|\xi\|}, \xi \in E_{*}
$$

Alors il n'existe pas de solution de classe $\mathscr{C}^{2}(\Sigma)$ de l'équation (5.11) ci-dessus. En effet, si une telle solution existe, on aura

$$
A^{a b} \tilde{D}_{a b} u \geq-v_{2}+(m-1)(1+v)-a(m-1)(1+v)^{\frac{3}{2}} .
$$

En un point $\xi \in \Sigma$ où $u$ atteint son maximum, on a : $v(\xi)=0$ et dans un repère $G$ orthonormé diagonalisant $\left(D_{a b} u(\xi)\right)$, (5.30) s'écrit au point $\xi$ sous la forme

$$
\sum_{1 \leq a \leq n+m-1} \tilde{D}_{a a} u \geq(m-1)(1-a) .
$$

Or $D_{a a} u(\xi) \leq 0$. Reportons dans (5.31), on voit que $1 \leq a$ ce qui est contradictoire.

On obtient la même conclusion s'il existe un réel $b>1$ tel que

$$
K(\xi) \geq \frac{b(m-1)}{\|\xi\|}, \xi \in E_{*}
$$

2- Considérons une fonction $K$ vérifiant les hypothèses du théorème 2 et supposons que pour tout $r \in\left[r_{1}, r_{2}\right]$, il existe $\xi \in \Sigma$ telle que

$$
K(r \xi) \neq(m-1) r^{-1}
$$


D'après la preuve du théorème 2 , il existe une solution $u_{1} \in \mathscr{C}^{\infty}(\Sigma)$ de l'équation (5.11) ci-dessus à gradient horizontal identiquement nul, $v_{2}\left(u_{1}\right)=0$, et telle que $r_{1} \leq e^{u_{1}} \leq r_{2}$. D'autre part, la preuve du théorème 3 donne une solution $u_{2}$ de cette même équation telle que $r_{1} \leq e^{u_{2}} \leq r_{2}$ et de sorte que pour un réel $\alpha>0$, assez grand, on ait :

$$
\mu_{a} e^{2 u_{2}} D_{a}^{a} u_{2}=-\alpha \sqrt{v\left(u_{2}\right)}
$$

Les deux graphes radiaux donnés par ces deux solutions ne sont pas homothétiques. Sinon, la fonction $u_{1}-u_{2}=$ Cste, et donc $v_{2}\left(u_{1}\right)=v_{2}\left(u_{2}\right)=0$. Reportons dans (5.33), et puisqu'alors $\mu_{a} e^{2 u_{2}} D_{a}^{a} u_{2}$ est identiquement nul, il en découle que $v_{1}\left(u_{2}\right)=0$, et par suite $u_{2}$ est une constante. Il existe alors $r \in\left[r_{1}, r_{2}\right]$ telle que $e^{u_{2}}=r$. L'équation (5.11) satisfaite par $u_{2}$ implique que $K(r \xi)=(m-1) r^{-1}$, quel que soit $\xi \in \Sigma$, contredisant ainsi (5.32).

\section{Références}

[1] S. Agmon : Lectures on Elliptic boundary value problems; Van Nostrand, Princeton, NJ 1965.

[2] I. Bakelman and B. Kantor : Existence of spherically homeomorphic hypersurfaces in euclidean space with prescribed mean curvature; Geometry and Topology, Leningrad, 1, 1974, 3-10.

[3] M. S. Berger : Nonlinearity and functional analysis ; Academic Press, New York, San Francisco, London 1977, Pure and Applied Mathematics 74.

[4] D. Gilbarg and N. Trüdinger : Elliptic partial differential equations of second order ; 2nd ed., Springer, New York, 1977.

[5] O.A. Ladyzhenskaya and N.N. Ural'tseva : Linear and quasilinear elliptic equations ; Academic press, New York and London, 1968.

[6] C. B. Morrey : Multiple integrals in the calculus of variations ; 130, Springer-verlag, Berlin, Heidelberg, New York, 1966.

[7] M. Nagumo : Degree of mapping in convex linear topological spaces; Amer. J. Math., 73, 1951, p. 497-511.

[8] A. E. Treibergs and S. W. Wei : Embedded hypersurface with prescribed mean curvature; J. Diff. Geo., 18, 1983, 513-521.

[9] K. Yano and S. Ishihara : Tangent and cotangent bundles; Marcel Dekker, New-York, 1973.

[10] K. Yano and S. Ishihara : Horizontal lifts of tensor fields and connections to tangent bundles; J. Math. and Mech., 16, 1967, 1015-1030. 